\title{
Lyman- $\alpha$ Forest and Cosmic Weak Lensing in a Warm Dark Matter Universe
}

\author{
Katarina Markovič ${ }^{1,4}$ and Matteo Viel $^{2,3}$ \\ ${ }^{1}$ University Observatory Munich, Ludwig-Maximilian University, Scheinerstr. 1, 81679 Munich, Germany \\ ${ }^{2}$ INAF - Osservatorio Astronomico di Trieste, Via G. B. Tiepolo 11, I-34131 Trieste, Italy \\ ${ }^{3}$ INFN/National Institute for Nuclear Physics, Via Valerio 2, I-34127 Trieste, Italy \\ ${ }^{4}$ Email: markovic@usm.lmu.de
}

(ReCEIVEd August 12, 2013; ACCEPTED November 20, 2013)

\begin{abstract}
We review the current state of the theory of large-scale structure in a warm dark matter (WDM) cosmological model. In particular, we focus on the non-linear modelling of the matter power spectrum and on the mass function of dark matter haloes. We describe the results of N-body simulations with WDM and mention the effects that could be induced by baryonic physics. We also examine the halo model of large-scale structure and its recently suggested modifications for a WDM cosmology, which account for the small-scale smoothness of the initial matter density field and better fit the results of N-body simulations. Having described the theoretical models, we discuss the current lower limits on the WDM particle mass, $m_{\mathrm{wdm}}$, which correspond to upper limits on the WDM temperature under the assumption that the particles are thermal relics. The best such constraints come from the Ly $\alpha$ forest and exclude all masses below $3.3 \mathrm{keV}$ at the $2 \sigma$ confidence level. We finally review the forecasts for future lensing surveys, which will be of the same order of magnitude as the already existing constraints from the Ly $\alpha$ forest data but explore a different redshift regime.
\end{abstract}

Keywords: cosmology: dark matter - cosmology: large-scale structure of universe - cosmology: theory - methods: numerical

\section{INTRODUCTION}

Here, we briefly outline how the idea of dark matter (DM) emerged and when warm dark matter (WDM) branched off the mainstream theory. The idea that the temperature of DM impacts the distribution of structure in the universe is as old as the notion that galaxies cluster on large scales.

An important step in DM history, which started with the measurements of displacements within spiral galaxies to measure their rotation, was to realise that the dynamical properties of galaxies and galaxy clusters did not seem to match their observed luminous mass (see e.g. Zwicky 1937; Schwarzschild 1954; Janák 1958; Abell 1962; Burbidge \& Sargent 1969; Paal 1976, and references therein). Moreover, thanks to the first Palomar Observatory Sky Survey (1949-1958, Reid et al. 1991), the distribution of galaxies in the sky was mapped for the first time in the mid $20^{\text {th }}$ century and showed that galaxies conglomerated not only into clusters, but also gave rise to the cosmological 'large-scale structure' (Press \& Schechter 1974; Rudnicki 1976; White \& Rees 1978; Jones \& Rees 1978; Wesson 1978; Aarseth
\& Fall 1980), whose properties depended on cosmological parameters.

It thus became clear early on that the so-called 'missing mass problem' (e.g. Faber \& Gallagher 1979) was unlikely to be solved by dark gaseous matter, that it had to be dark stars, black holes, comets, or something else, like the conveniently weakly interacting massive particle - the neutrino. Assuming the missing mass was made up of massive neutrinos and other weakly interacting particles, an upper limit of $8 \mathrm{eV} \mathrm{c}^{-2}$ could be placed on their masses, assuming the measured expansion of the universe ${ }^{1}$ (Cowsik \& McClelland 1972).

The discovery of the cosmic microwave background (CMB) by Penzias \& Wilson (1965); Dicke et al. (1965) resulted in the acceptance of the Hot Big Bang origin of the universe. Big Bang Nucleosynthesis (BBN) put severe constraints on the amount of baryonic matter in the universe $\left(\Omega_{\mathrm{b}} \ll 1\right.$, Schramm \& Wagoner 1977; Olive, Steigman \& Walker 2000, and references therein), and, combined with

\footnotetext{
${ }^{1}$ This was consistent with the measurement of neutrino mass from the Coma cluster density profile of Cowsik \& McClelland (1973).
} 
the requirement that the total density in the universe was close to the critical density for flatness, ${ }^{2}$ gradually led to the belief that DM is made of new elementary particles.

One of the first candidates was a massive neutrino, but more general new particles were considered quickly, like other stable neutral leptons (Gunn et al. 1978; Tremaine \& Gunn 1979). The theory of supersymmety (SUSY) offered new candidate particles, like the gravitino with a $\mathrm{keV}$ mass (Pagels \& Primack 1982), or the photino (Sciama 1983). In addition the axion, whose Jeans Mass would be smaller than galactic scales, was considered as a DM candidate (Stecker \& Shafi 1983; Shafi \& Stecker 1984). These particles were distributed in the universe as a perturbed density field, which became gravitationally coupled to the density field of baryons in the matter dominated era. However, because there was no energy dissipation for the particles of DM, they could not condense into bound objects easily. This was especially true for massive neutrinos with masses $\sim \mathrm{eV}$, whose damping scales reached the sizes of galaxy clusters and even superclusters (Bond, Efstathiou \& Silk 1980; Schramm \& Steigman 1981). This meant that if the DM that was closing the universe was made up of massive neutrinos, the distributions of dark and luminous matter would have to be very different (Bruns \& Zinnecker 1983). In fact, in such a neutrino-dominated hot dark matter (HDM) model, the mechanism for galaxy formation was considered to be a fragmentation of large objects, which collapse first, as proposed by Zeldovich (1970). These objects, which collapsed along one dimension first, were known as 'pancakes'. After collapsing along two dimensions, they became filaments and finally spherically symmetric DM haloes.

The observations of the dynamics of galaxies, galaxy clusters, and superclusters were compared to the amount of visible mass by many authors (e.g. Bruns \& Zinnecker 1983, and references therein). A trend seemed to emerge, where the 'missing mass' fraction increased with object size, implying that the relation between the distributions of dark and luminous matter densities must be more biased on large scales.

Simultaneously, hierarchical merging of structure was considered via N-body simulations of the formation of the largescale structure in the universe (Aarseth \& Fall 1980; White \& Negroponte 1982). In this picture, structure formed as a consequence of pure gravitational collapse of the initial linear density perturbations. Such 'bottom-up' hierarchical structure formation occurred if the matter density in the universe was dominated by particles more massive than at least several tens of eV (Bond, Szalay \& Turner 1982), but it was not possible for the $\mathrm{m}_{v} \sim 30 \mathrm{eV}$ neutrinos (Peebles 1982). The bottom-up scenario became strongly favoured in 1984, since observations of dwarf galaxies as well as those of largescale structure overall put strong lower limits on the mass of the DM particle (Lin \& Faber 1983; Kaiser 1983; Madsen

\footnotetext{
${ }^{2}$ Flatness implies that the energy density in the universe is equal to the "critical density' for flatness, i.e. the total density parameter, $\Omega \sim 1$, measured from the Hubble parameter and assumed from 'naturalness' of zero spatial curvature, $\kappa=0$ of the Einstein-de Sitter model.
}

\& Epstein 1984). In other words, the standard model of DM became the cold dark matter (CDM) model.

However, Klypin et al. (1999) noticed a discrepancy in the observed numbers of the smallest galaxies, assumed to reside within DM haloes with masses $M_{\text {dwarf }} \leq 10^{9} M_{\odot}$ and what they expected these numbers to be from running their numerical simulations of structure formation with CDM. They proposed that the numerical simulations might be modified to account for this discrepancy, which has become known as the $d$ warf galaxy problem or missing satellite problem (Bode, Ostriker, \& Turok 2001), since the number of small objects observed fell significantly short of the expectation.

In recent years, N-body and hydro-dynamical codes have improved significantly in matching the small scales of $\Lambda \mathrm{CDM}$ to observations, for example via the suppression of the formation of baryonic objects within small DM haloes (e.g. 'cosmic web stripping', Benitez-Llambay et al. 2012). In addition, surveys like the Sloan Digital Sky Survey have found new dwarf galaxies around the Milky Way (see Bullock 2010, and references within for a review of the problem).

It is possible that this small-scale crisis of $\Lambda \mathrm{CDM}$ could be solved or alleviated with better numerical prescriptions for the complex baryonic processes (see e.g. Brooks et al. 2012) or it could be due to observational biases. However, the density profiles and concentrations of individual haloes (Donato et al. 2009) as well as the properties of voids (Tikhonov et al. 2009) do not seem to match what one would expect from pure $\Lambda \mathrm{CDM}$ model. Baryonic processes are difficult to invoke for explaining the properties of dwarf galaxies that are DM dominated, making it hard to fit their observational properties.

These long known 'missing satellite' and 'core-cusp problems' are related to more recently defined 'too-big-to fail' problem, being that the most massive Milky Way subhaloes from local $\Lambda \mathrm{CDM}$ simulations do not have dynamical properties similar to the observed Milky Way dwarf galaxies. For a recent review of the small-scale issues of $\Lambda \mathrm{CDM}$ see Weinberg et al. (2013).

An elegant solution has been considered in the past, which has introduced WDM in a simple $\Lambda$ WDM model with one additional parameter, which could explain all or some of these discrepancies. Because of its free-streaming, WDM is capable of damping the density field on small scales without any change to the large-scale behaviour of structure or to the dynamical evolution of space-time. For this reason we now discuss and summarise how to calculate non-linear corrections to predict the statistical properties of cosmological structure. This is not a straightforward task, but nonetheless, we describe attempts to develop a prescription valid also in $\Lambda \mathrm{WDM}$ models that may be used one day to account for some of the discrepancies at small scales of $\Lambda \mathrm{CDM}$.

However, it should be noted that there exists a phasespace density imposed lower bound on the fermionic DM particle mass, called the Tremaine-Gunn bound (Tremaine \& Gunn 1979; Hogan 1999), due to which it may not be possible for a WDM model to produce the relatively large 
cores that we seem to observe. Large halo cores can namely only be produced by very small particle mass, $m_{\mathrm{wdm}}$ (Shao et al. 2013). This has become known as the 'too-small-tosucceed' problem and put significant pressure on the WDM scenario.

In this review, we discuss the existing literature on constraining the WDM particle mass, $m_{\mathrm{wdm}}$, using the statistical properties of the large-scale structure. We choose this approach in hope that it may contain some information not contaminated by the uncertainties arising out of a lack of a rigorous model of baryonic feedback and cooling processes. There are many other works that look at individual objects of the large-scale structure and hope to constrain WDM from their properties (e.g. Pacucci, Mesinger, \& Haiman 2013; Lovell et al. 2012; Maccio et al. 2012; Vinas, Salvador-Sole, \& Manrique 2012; Sommer-Larsen \& Dolgov 2001).

In particular, we discuss in some detail the modelling of non-linear large-scale structure needed for comparison with data. We choose two observables to constrain our models: the Ly $\alpha$ forest and the cosmic shear (weak lensing) power spectrum, both of which require an accurate modelling of the non-linear matter power as a first step in the modelling of their basic properties. In Section 2, we summarise the general background physics of the smoothing of the linear matter density field by the free-streaming DM and the calculation of the linear matter power spectrum. We also briefly discuss the particle candidates for WDM. We then describe prescriptions for calculating the non-linear matter power spectra in the WDM scenario. In particular, we discuss N-body simulations, the halo model, and the current status of the two approaches for calculating the statistics of the large scales in the universe. We summarise the current limits coming from the Ly $\alpha$ forest data in Section 3, which present the strongest constraints on the temperature of DM to date. Finally, we report on forecasts that have been made on the WDM temperature obtained from future weak lensing surveys like Euclid ${ }^{3}$ in Section 3.2.

\section{GENERAL FRAMEWORK}

Neutrinos decouple when the temperature of the primordial plasma is $T \sim 1 \mathrm{MeV}$ and $a \sim 10^{-10}$ and become nonrelativistic when $T_{\mathrm{h}} \sim m_{\mathrm{h}} / 3 k_{\mathrm{B}} \cdot{ }^{4} \mathrm{DM}$ decouples and becomes non-relativistic much earlier in both the CDM and WDM cases. If WDM has a simple thermal history, analogous to neutrinos, but with a larger particle mass, we can calculate its free-streaming. Such a DM particle is called a thermal relic, because it was once in equilibrium with itself.

The Jeans length can be calculated for a perfect fluid and denotes the limit on which the gravitational effect balance out the thermal effects (Bond \& Szalay 1983). For collisionless fluids like the DM and neutrino fluids, we can define the analogous comoving free-streaming wavenumber, which

\footnotetext{
${ }^{3}$ Amendola et al. (2012); Refregier et al. (2010)

${ }^{4}$ The Boltzmann constant, $k_{\mathrm{B}}=8.617 \times 10^{-5} \mathrm{eV} \mathrm{K}^{-1}$.
}

PASA, 31, e006 (2014)

doi: $10.1017 /$ pasa.2013.43 tells how far the fast-moving particles can travel within the gravitational time-scale, i.e. in the time of free-fall (Boyarsky et al. 2009a):

$$
k_{\mathrm{fs}}(a)=\sqrt{\frac{3}{2}} \frac{a H(a)}{v_{x, \text { median }}}
$$

where $v_{x}=1$, when the particles are relativistic. When they go non-relativistic (i.e. when $3 k_{\mathrm{B}} T_{0, x} \lesssim m_{x}$ ),

$$
v_{x}=\frac{3 k_{\mathrm{B}} T_{0, x}}{a m_{x}}
$$

and then $k_{\mathrm{fs}} \rightarrow \infty$ as $a \rightarrow 1$ and $T \rightarrow 0$, which is the case for CDM very early on, and therefore the effects of freestreaming are pushed to very very large $k$, i.e. extremely small scales. This means that the damping of the overdensity field becomes insignificant at cosmological scales.

In fact, even in mixed DM models (C+HDM), where the HDM component makes up a small fraction of the total energy density as in the $\Lambda \mathrm{CDM}+$ neutrinos, $v \Lambda \mathrm{CDM}$, the perturbations in the cold component are modified by the free-streaming of the HDM. In this scenario the larger scales suffer more free-streaming damping and therefore the perturbations in the HDM cannot grow until late times, which gravitationally affects the perturbations in the cold component, slowing down the growth of the perturbation amplitudes (Primack \& Gross 1998; Ghigna et al. 1997; Klypin et al. 1995; Nolthenius, Klypin \& Primack 1994; Klypin et al. 1993; Gawiser \& Silk 1998; Primack 1997; Primack et al. 1995; Ma \& Bertschinger 1995; Zentner \& Bullock 2003; Primack 2003).

The most basic model of WDM particles is to assume that they are thermal relics. This means that they were in thermal equilibrium at some point. When their temperature and density dropped, they went out of equilibrium (e.g. Bond \& Szalay 1983) and DM particles decoupled from each other. Instead, the sterile neutrino particles, that will be discussed later, were never in thermal equilibrium.

Theoretically there would have been another kind of decoupling. This would have been when DM particles and baryons were in an extremely dense environment and so there would have been a significant interaction rate between them. We know very little about this regime, because we would have to know the mass and interaction cross-section of DM particles, but we do not even know the nature of the interaction (if any) between DM particles and other types of matter.

However, it is most likely that these two decouplings happened at the same time, because any self-interaction of DM is likely to involve the weak, strong, or electromagnetic force, which means this self-interaction would necessarily involve baryons. Were this not the case, it may be that the interaction between baryons and DM particles is weaker than the interaction among DM particles. In this case the decoupling from baryons would happen at an earlier time than decoupling of DM out of equilibrium. 
The smallest important scale feature in the linear matter power spectrum is the suppression induced by DM freestreaming. In the WDM model the scale of suppression is called the free-streaming scale, $k_{\mathrm{fs}}$, and corresponds to the mode that enters the horizon at the time when WDM particles become non-relativistic, $t_{\text {rel }}$. A species can become non-relativistic while still in thermal equilibrium or after it decouples (Bond \& Szalay 1983; Bode et al. 2001; White et al. 1987). If it is after, we say that DM particles decouple while non-relativistic. This is what we often assume in modelling the large-scale structure in these models, for the sake of simplicity.

From Bond \& Szalay (1983), we get the temperature of WDM relative to that of the photons, from which one can calculate the total WDM density for a particular particle model (giving $g_{\mathrm{wdm}}$ and $m_{\mathrm{wdm}}$ ):

$$
\Omega_{\mathrm{wdm}}=\frac{1.1}{h^{2}}\left(\frac{100}{3.9}\right)\left(\frac{g_{\mathrm{wdm}}}{1.5}\right)\left(\frac{T_{\mathrm{wdm}}}{T_{\gamma}}\right)^{3}\left(\frac{m_{\mathrm{wdm}}}{1 \mathrm{keV}}\right),
$$

where $g_{*}=3.9\left(T_{\gamma} / T_{\mathrm{wdm}}\right)^{3}$ is the number of all relativistic degrees of freedom at WDM decoupling, $g_{\text {wdm }}$ are the degrees of freedom for the WDM, $T_{\gamma}$ is the present day photon temperature, and $T_{\mathrm{wdm}}$ is the temperature of WDM. We can calculate the degrees of freedom:

$$
g_{\mathrm{wdm}}= \begin{cases}N_{\mathrm{wdm}} & \text { bosons } \\ \frac{3}{4} N_{\mathrm{wdm}} & \text { fermions }\end{cases}
$$

where $N_{\mathrm{wdm}}$ are the number of spin degrees of freedom. Then assuming $\Omega_{\mathrm{dm}}=\Omega_{\mathrm{wdm}}$ gives a direct relationship between $T_{\mathrm{wdm}}$ and $m_{\mathrm{wdm}}$. Otherwise we must introduce a new parameter $f_{\mathrm{wdm}}=\Omega_{\mathrm{wdm}} / \Omega_{\mathrm{dm}}$, the fraction of WDM. This parameter becomes relevant when we start to consider C+WDM models.

In addition we can calculate the velocity dispersion of WDM particles relative to that of the neutrinos (Bond et al. 1980):

$$
\sqrt{\left\langle v^{2}\right\rangle_{v}}=6 \mathrm{kms}^{-1}\left(\frac{30 \mathrm{eV}}{m_{v}}\right)(1+z)
$$

Rescaling for WDM, if it has decoupled while relativistic:

$$
\sqrt{\left\langle v^{2}\right\rangle_{\mathrm{wdm}}}=\sqrt{\left\langle v^{2}\right\rangle_{v}}\left(\frac{T_{\mathrm{wdm}}}{m_{\mathrm{wdm}}}\right)\left(\frac{m_{v}}{T_{v}}\right) .
$$

If particles decouple while non-relativistic, $\sqrt{\left\langle v^{2}\right\rangle_{\text {wdm }}} \lesssim$ $\mathrm{cm} \mathrm{s}^{-1}$, so any further damping is insignificant and the species becomes effectively 'cold'.

\subsection{The linear power spectrum}

In the case of WDM, the initial matter power spectrum emerging from radiation domination is modified by an additional transfer function due to free streaming. Viel et al. (2005) used a fitting function that can however be calculated very accurately with a numerical Boltzmann equation solver code, like for example CMBFAST (Seljak \& Zaldarriaga 1996), CAMB (Lewis, Challinor \& Lasenby 2000), or CLASS (Blas, Lesgourgues, \& Tram 2011; Lesgourgues 2011; Lesgourgues \& Tram 2011). These codes solve the equations describing the growth of perturbations in the radiation dominated universe in a semi-analytic line-of-sight approach.

The fitting function of Viel et al. (2005) with $v=1.12$ (the alternative is $v=1.2$ like in Bode et al. 2001) contains a scale-break parameter, which is used in calculating the linear matter power spectrum by multiplying with the following WDM transfer function:

$$
\begin{aligned}
& \mathcal{T}_{\text {wdm }}(k)=\left(1+(\alpha k)^{2 v}\right)^{-5 / v} \text { and so } \\
& P_{\text {wdm }}^{\text {lin }}(k)=P_{\text {cdm }}^{\text {lin }}(k) \mathcal{T}_{\text {wdm }}^{2}(k),
\end{aligned}
$$

where the scale breaks at:

$$
\alpha=0.049\left(\frac{m_{\mathrm{wdm}}}{1 \mathrm{keV}}\right)^{-1.11}\left(\frac{\Omega_{\mathrm{wdm}}}{0.25}\right)^{0.11}\left(\frac{h}{0.7}\right)^{1.22} .
$$

Alternatively, Boyanovsky, de Vega \& Sanchez (2008b) found a transfer function for a general initial thermal distribution of DM particles - cold WIMP DM, thermal fermionic, or bosonic DM.

The linear power spectrum, $P_{\mathrm{wdm}}^{\text {lin }}(k)$, must then be normalised to ensure the value $\sigma^{2}{ }_{8}$ at $R=8 h^{-1} \mathrm{Mpc}$. Finally we now can plot the linear matter power spectra in Figure 1. The lightest WDM particle mass shown $(500 \mathrm{eV})$ causes the linear theory matter power spectrum to be suppressed dramatically at a wavenumber significantly above $1 \mathrm{hMpc}^{-1}$ . The matter power spectrum of WDM starts to turn off well above the free-streaming scale, which changes the slope of the power spectrum to fall much more steeply than $n_{\text {eff }}=\log$ $P(k) / \log k=-3$, which is the slope for standard, bottomup structure formation (White \& Frenk 1991; Knebe et al. 2003).

In fact the above seemingly artificial scale break, $\alpha$, relates to the free-streaming length of thermal relic WDM particles (Zentner \& Bullock 2003):

$$
\lambda_{\mathrm{fs}} \simeq 0.11\left[\frac{\Omega_{\mathrm{wdm}} h^{2}}{0.15}\right]^{1 / 3}\left[\frac{m_{\mathrm{wdm}}}{\mathrm{keV}}\right]^{-4 / 3} \mathrm{Mpc},
$$

which of course is related to the Fourier space free-streaming scale, where free-streaming length effect contribute most to the power (see also Equation (1)):

$$
k_{\mathrm{fs}} \sim 5 \mathrm{Mpc}\left(\frac{m_{\mathrm{wdm}}}{\mathrm{keV}}\right)\left(\frac{T_{v}}{T_{\mathrm{wdm}}}\right) .
$$

We plot $k_{\mathrm{fs}} / 10$ in Figure 1, because it is the scale around which the significant suppression of the power in the linear matter power spectrum begins. It is an interesting open-ended question why the free-streaming suppression reaches scales so much larger than the free-streaming scale. This has been explored, among others, by Smith \& Markovič (2011). 

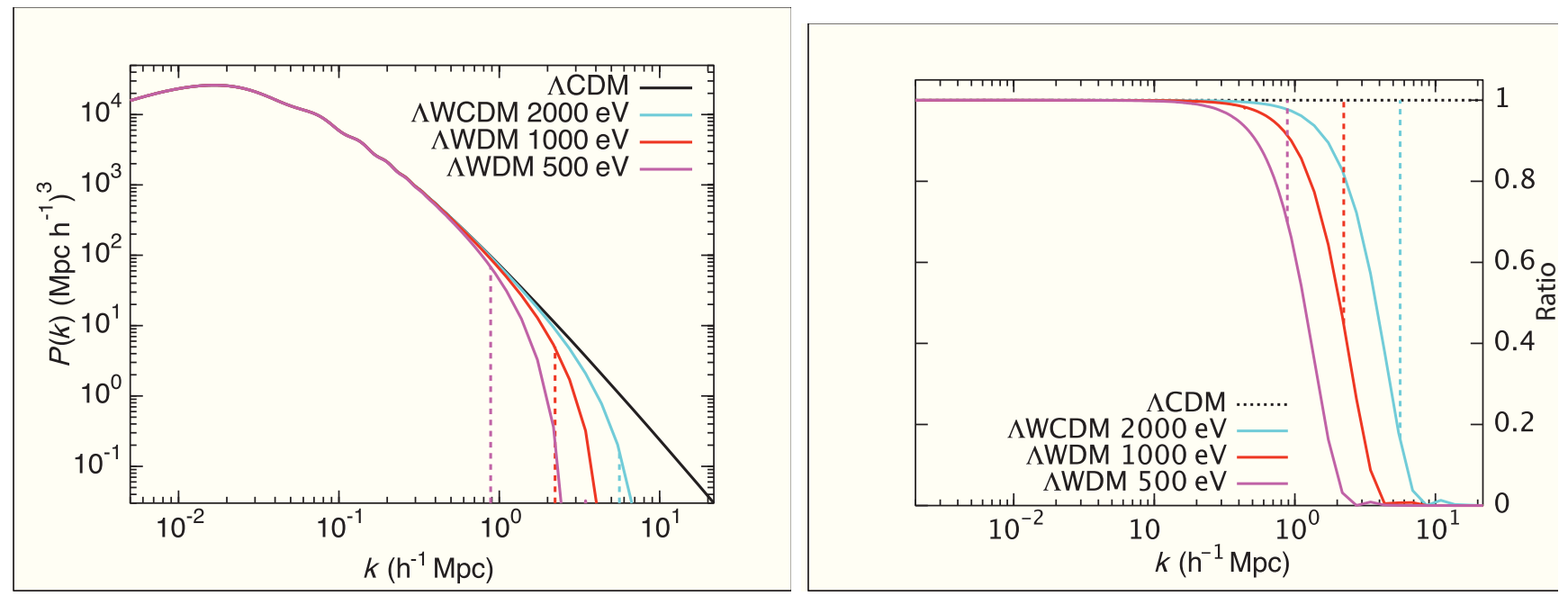

Figure 1. Left: The linear matter power spectra for three different WDM models and standard CDM. The particle masses, $m_{\mathrm{wdm}} \in\{0.5,1.0,2.0\} \mathrm{keV}$, are colour coded with magenta, red, and cyan, respectively. The vertical lines correspond to a tenth of the free-streaming wavenumber, $k_{\mathrm{fs}} / 10$, for each model of WDM. These power spectra were produced using the Boltzmann solver CLASS (Lesgourgues 2011).

Right: ratios between the WDM and CDM power spectra, $P_{\mathrm{wdm}}(k) / P_{\mathrm{cdm}}(k)$, to clearly see the suppression with respect to the $k_{\mathrm{fs}} / 10$.

In addition, we can define a corresponding mass found, on average, in a volume with such a radius or free-streaming mass:

$$
M_{\mathrm{fs}}=\frac{4 \pi \rho_{\mathrm{m}}}{3}\left(\frac{\lambda_{\mathrm{fs}}}{2}\right)^{3}
$$

where $\rho_{\mathrm{m}}$ is the comoving background matter density and $\lambda_{\mathrm{fs}}$ is the comoving free-streaming length defined in Equation (9) (note that different definitions for the free-streaming mass are used in the literature). We will come back on this issue in Section 2.3.2.

In Figure 2, we plot the linear matter power spectrum alongside the CMB power spectrum for the HDM scenario to illustrate the impact on the CMB and matter power of such a small mass candidate; it is thus clear that heavier masses will impact much less on these two observables at the largest scales. We plot the power spectra in the neutrinolike (but with $m \sim 10 \mathrm{eV}$ ) scenario being all of DM. We expect the power spectra to be suppressed at very large $l$ due to the free-streaming effect and to increase for small $l$, due to a mixture of two effects: changes in the matterradiation equality and because the primordial power spectrum is normalised at $k=0.05 \mathrm{hMpc}^{-1}$ causing the smallscale-suppressed power spectrum to be boosted on large scales.

Since, $l=2000$ corresponds to about a $k \sim 0.2 \mathrm{hMpc}^{-1}$ at $z_{\mathrm{CMB}}=1000$, we expect the effect on the $\mathrm{CMB}$ from reasonable WDM scenarios to be completely negligible. In the right panel of Figure 1 it can be noted that at $k=0.2$, $h \mathrm{Mpc}^{-1}$, the suppression is clearly less than $1 \%$ for $m_{\mathrm{wdm}}=$ $1 \mathrm{keV}$.

\subsection{Sterile neutrinos}

We have discussed some of the particle candidates for DM in this introductory section of this review. A further hypothetical particle that has sparked interest is the sterile neutrino, which does not require an extension of the Standard Model with SUSY (Dodelson \& Widrow 1994; Fuller et al. 2003; Asaka, Blanchet \& Shaposhnikov 2005; Abazajian 2006; Boyarsky et al. 2006; Petraki \& Kusenko 2008; Laine \& Shaposhnikov 2008; Kusenko 2009; Hamann et al. 2010; Boyarsky, Iakubovskyi \& Ruchayskiy 2012). Sterile neutrinos are assumed to be singlet right-handed particles that become relatively heavy compared to standard, active neutrinos, which receive small masses via a seesaw mechanism (Dodelson \& Widrow 1994). The lightest of the additional neutrinos can then have a mass in the keVrange, meaning that it resembles a WDM. However, the sterile neutrinos are assumed to never have been in thermal equilibrium; therefore, their abundance was suppressed.

In the above calculation of the free-streaming scale we have used three parameters that describe the thermal relic WDM model: the particle mass, $m_{\mathrm{wdm}}$, the energy density, $\Omega_{\mathrm{wdm}}$, and the temperature $T_{\mathrm{wdm}}$, where the degrees of freedom at WDM decoupling, $g_{\mathrm{wdm}}^{*}$, are determined solely by the particle mass and its energy density. We can conveniently re-parametrise the particle mass of the neverthermalised sterile neutrino in terms of the thermal relic mass, such that they are interchangeable in the calculation of the impact of their free-streaming on the large-scale structure:

$$
m_{v \mathrm{~s}}=4.43\left(\frac{m_{\mathrm{wdm}}}{\mathrm{keV}}\right)^{4 / 3}\left(\frac{\omega_{\mathrm{wdm}}}{0.1225}\right)^{-1 / 3} \mathrm{keV}
$$



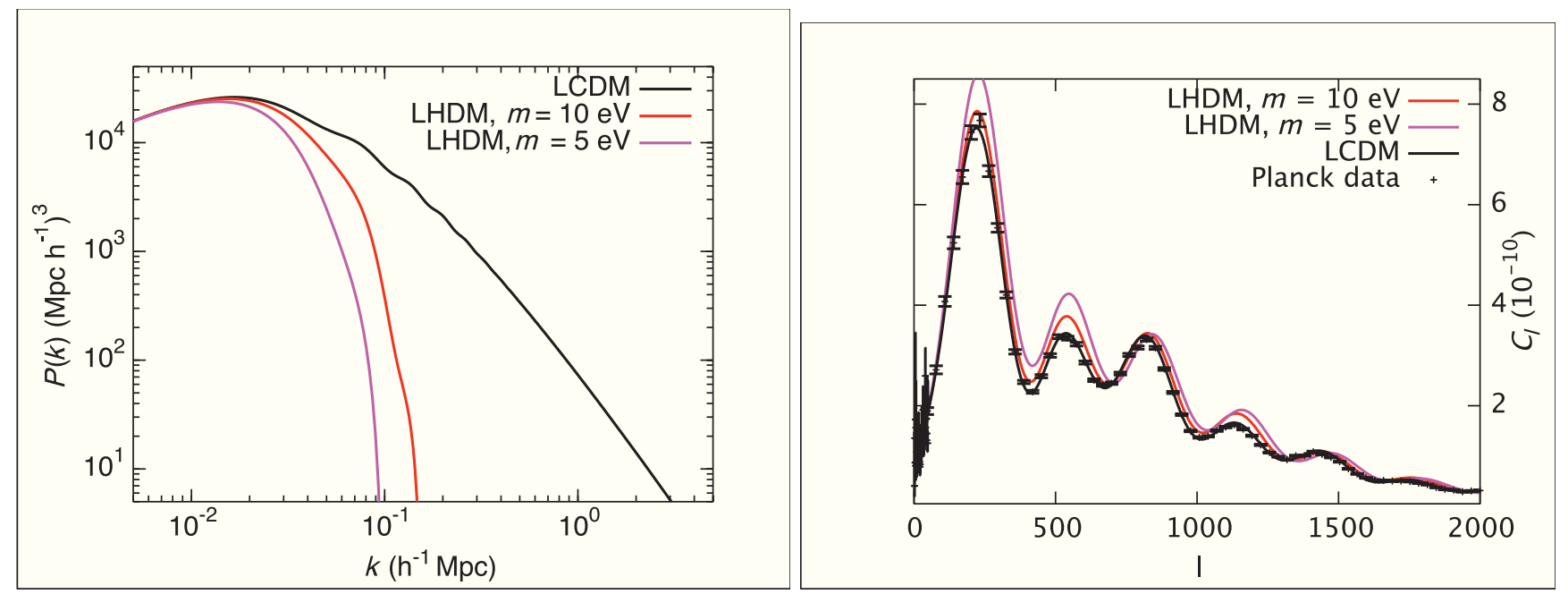

Figure 2. We plot the effect of a hot thermal relic particle (hotter than a WDM candidate) on the cosmic microwave background $C(l)$ 's: we show models that have been long ruled out, where all the DM is made up of very light, neutrino-like particles with masses of $m_{\mathrm{wdm}}=0.005$ and $0.01 \mathrm{keV}$.

Left: The three-dimensional linear matter power spectrum, $P(k)$, for CDM (black) and the two 'HDM' models (magenta and red, respectively). Compare this extreme case to the more plausible models in Figure 1 that will impact at a much smaller level. Right: The CMB un-lensed $C(l)$ 's in the corresponding HDM models. The cosmological model is the Planck best fit as in the rest of the paper, the error bars are those of Planck survey (Planck Collaboration et al. 2013).

where $\omega_{\mathrm{wdm}}=\Omega_{\mathrm{wdm}} h^{2}$ (Viel et al. 2005). ${ }^{5}$ In this situation the degrees-of-freedom are fixed and abundance depends on the mass and energy density of sterile neutrinos. Note that the above relation between thermal and sterile neutrino masses is valid only for the so-called non-resonant production mechanism (Dodelson \& Widrow 1994). When other mechanisms are involved (e.g. resonant production; Boyarsky et al. 2009 b), the relation is non-trivial.

\subsection{The non-linear power spectrum}

Now that we have shown the effects of WDM on the linear matter density field we must outline some tools for the standard model of structure formation. In the matter dominated era, the density contrast grows and eventually reaches unity, where non-linearities must be properly accounted for and modelled. From this point on, standard perturbation theory is no longer appropriate and we must employ approximation methods as exact solutions to the Einstein field equations no longer exist.

It is necessary to have a robust model of non-linear structure in order to take full advantage of future weak lensing data. For this reason we compare the non-linear matter power spectra extracted from simulations with derived non-linear models. The halo model of non-linear structure is based on the assumption that large-scale structure is made up of individual objects occupying peaks in the matter over-density field (Press \& Schechter 1974; Seljak 2000; Cooray \& Sheth 2002). The most important elements of this model, the mass function, the halo bias (Press \& Schechter 1974), and the halo density profile (Navarro, Frenk, \& White 1997), are based on the assumptions that all DM in the universe is found in

\footnotetext{
${ }^{5}$ with $h=H_{0} / 100 \mathrm{~km} \mathrm{~s}^{-1} \mathrm{Mpc}^{-1}$, the Hubble parameter
}

haloes and that there is no observable suppression of smallscale over-densities from early-times free-streaming of DM particles or late-times thermal velocities.

These are characteristic properties of CDM, but do not apply to WDM. For this reason one should re-visit the modelling of cosmological structure. Smith \& Markovič (2011); Schneider et al. (2012); Dunstan et al. (2011); Schneider, Smith, \& Reed (2013b); and Angulo, Hahn, \& Abel (2013) modified the halo model by applying a specific prescription to the non-linear contribution, in addition to suppressing the initial density field, modelled by applying a transfer function from Equation (7) to the linear matter power spectrum. We discuss the halo model in Section 2.3.2. However, we wish to first summarise some results from N-body simulations.

\subsubsection{WDM simulations}

$\mathrm{N}$-body simulations have long been considered important in calculating the properties of the large-scale structure of DM and the formation of this structure in the CDM scenario (Appel 1985; Barnes \& Hut 1986; Katz, Weinberg \& Hernquist 1996; Frigo 1999; Bagla \& Ray 2003; Springel 2005). In recent years, this method has also been applied to the WDM case (Boehm et al. 2005; Boyanovsky, de Vega \& Sanchez 2008a; Wang \& White 2007; Zavala et al. 2009; Colombi et al. 2008; Viel et al. 2012; Schneider et al. 2012; Dunstan et al. 2011; Benson et al. 2012; Semenov et al. 2013). Numerical convergence for WDM (and HDM) simulations is particularly difficult to achieve as pointed out by Wang \& White (2007). The reason is due to the spurious fragmentation of filaments that give rise to a pattern of small mass haloes. This effect can be alleviated by increasing the number of particles (although convergence 

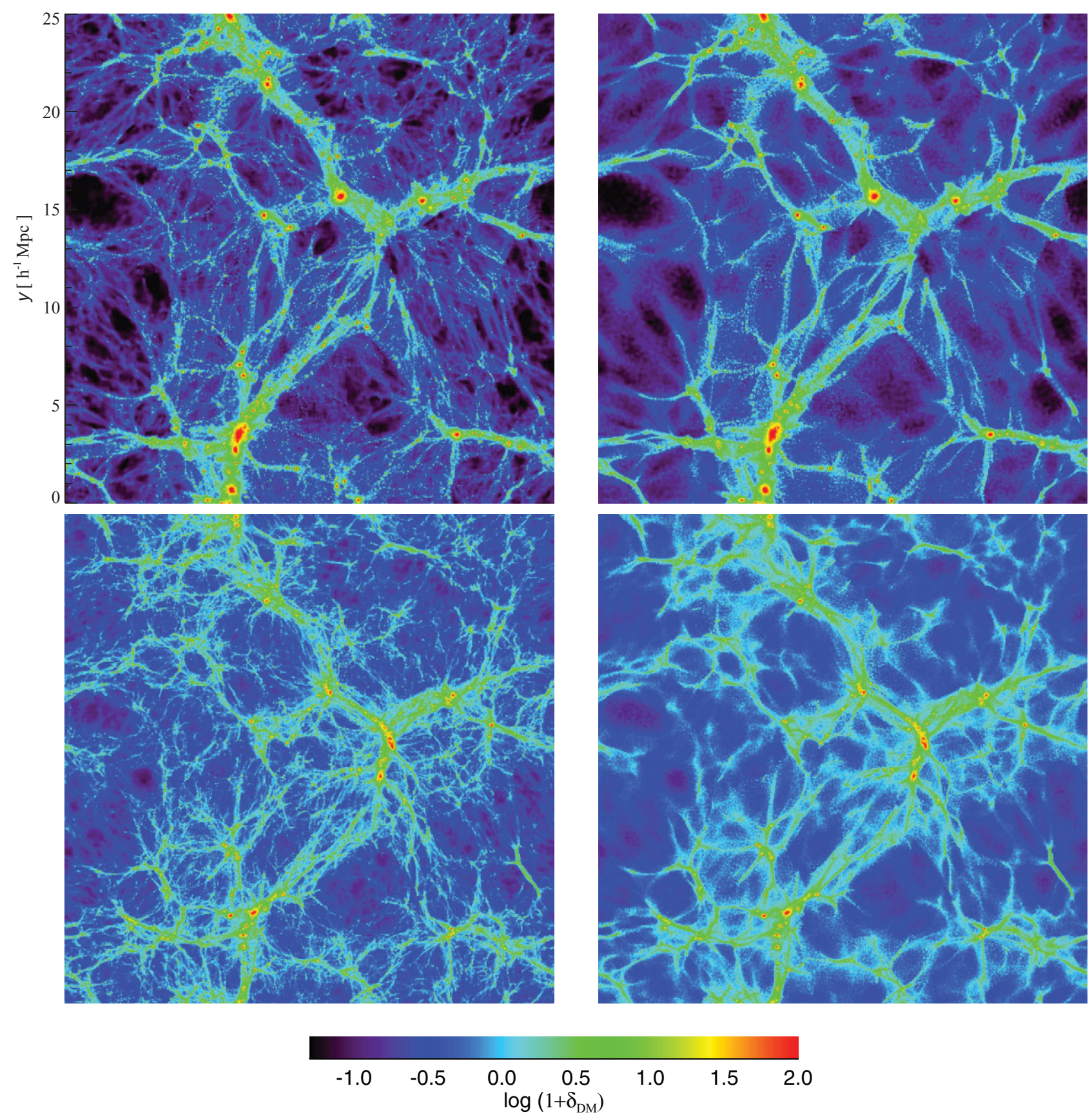

Figure 3. We plot the projected DM overdensity from a high-resolution hydro-dynamical simulation of Viel et al. (2012), at redshifts $z=0$ (upper panels) and $z=2$ (lower panels) for $\Lambda \mathrm{CDM}$ and $1 \mathrm{keV}$ WDM, in the left and right columns, respectively. The amount of substructure present in the $\Lambda \mathrm{CDM}$ model is more pronounced with respect to the WDM one. The box size is 25 comoving $\mathrm{Mpc}^{-1}$ and the thickness is 5 comoving Mpc $\mathrm{h}^{-1}$.

is slow) or by preventing the fragmentation of such structures. In any case, convergence tests of the relevant simulated physical quantities (like the Ly $\alpha$ forest flux and/or the mass function or matter power spectrum) must be performed in order not to be affected by this at the scales or redshifts of interest. A post hoc solution was proposed by Schneider et al. (2013b), which does not solve the problem, but corrects the result via subtraction of spurious haloes, while Lovell et al. (2013) identifies spurious haloes in the initial conditions.
$\mathrm{N}$-body simulations assume that collapsing matter is nonrelativistic $(\rho \gg P)$ and that collapse is only possible on sub-horizon scales $(k \gg a H)$. Therefore in $\Lambda \mathrm{CDM}$, the nonrelativistic, Newtonian perturbation equations are sufficient and so, the collisionless Boltzmann and Poisson equation are solved in a discreet way.

These equations are normally solved by an N-body code, e.g. GADGET (Springel, Yoshida \& White 2001). It is difficult to achieve this simply with finite difference methods, so Monte-Carlo-like N-body simulations are employed to 
Markovič and Viel
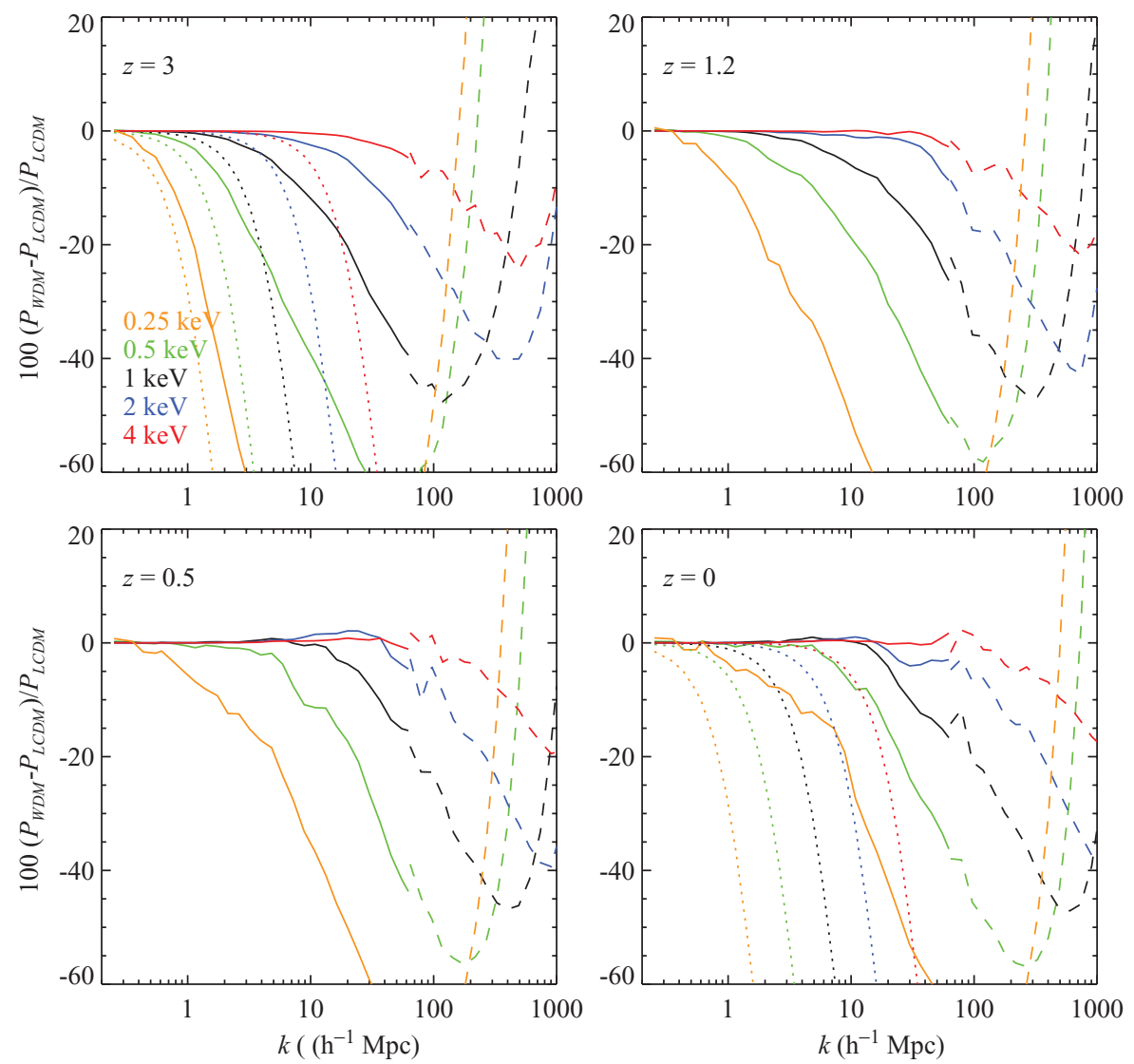

Figure 4. Percentage differences between WDM and CDM non-linear matter power spectra from hydrodynamical simulations at high-resolution. The solid lines show the large-scale power, while the dashed lines describe the small-scale power obtained with the folding method in order to reach smaller scales (see Jenkins et al. 1998; Colombi et al. 2008, for details). The dotted line is the suppression to the linear matter power spectrum and is the same both in the $z=3$ and $z=0$ panels. The different panels show $z=0,0.5$, $1.2,3$. Note that the steep rise on scales, $k>50 \mathrm{hMpc}^{-1}$, is affected by the poor resolution of the WDM simulations and it is not fully physical (although an increase of power could be expected and it might be due to the different DM density profile at small scales).

integrate the Boltzmann equations of $\mathrm{N}$ particles populating the phase space, using the method of characteristics. ${ }^{6}$

Smith et al. (2003) compared the standard CDM halo model to CDM simulations of large-scale structure formation and developed an analytical fit to the non-linear corrections of the matter power spectrum, known as HALOFIT. Markovič et al. (2011) and Viel et al. (2012) applied these corrections to a linear matter power suppressed by the Viel et al. (2005) WDM transfer function (Equation 7). Viel et al. (2012) ran cosmological N-body simulations (DM only) in the WDM scenario (see Figure 3). They found that the WDM

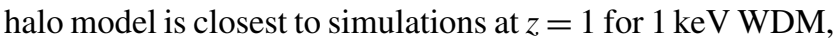
but that it over-estimates the suppression effect at $z=0.5$ for $0.5 \mathrm{keV}$ WDM by about $5 \%$ on scales $k>1 \mathrm{hMpc}^{-1}$. On scales $k<1 h_{\mathrm{Mpc}^{-1}}$ however, the HALOFIT non-linear cor-

\footnotetext{
${ }^{6}$ The method of characteristics is a way of solving partial differential equations by reducing them to a set of ordinary differential equations and integrating from a set of initial conditions. In other words the partial differential equations are solved by integration along characteristic curves, in this case the characteristic curves of the collisionless Boltzmann equation.
}

rection describes the simulations better than the halo model, even though on smaller scales it severely underestimates the suppression effect, which becomes worse at lower redshifts. A further small modification of the WDM halo model improves its correspondence to the simulations and allows one to use it at small scales (Section 2.3.2).

Viel et al. (2012) consider varying resolutions and WDM models. These simulations were run using the N-body code GADGET-2, for which the initial conditions were generated using the WDM-suppressed linear matter power spectrum in Equation (7). In Figure 4 we see plotted the percentage differences between the WDM and CDM non-linear matter power spectra for several different WDM models, denoted by the different thermal relic particle masses. This plot shows the suppression effect growing not only with decreasing particle mass (i.e. increasing WDM temperature), but also with increasing redshift and demonstrates the effect that the WDM signal is erased with time due to the non-linear growth of structure. It may be noted that the free-streaming scale below which the power spectrum becomes exponentially 

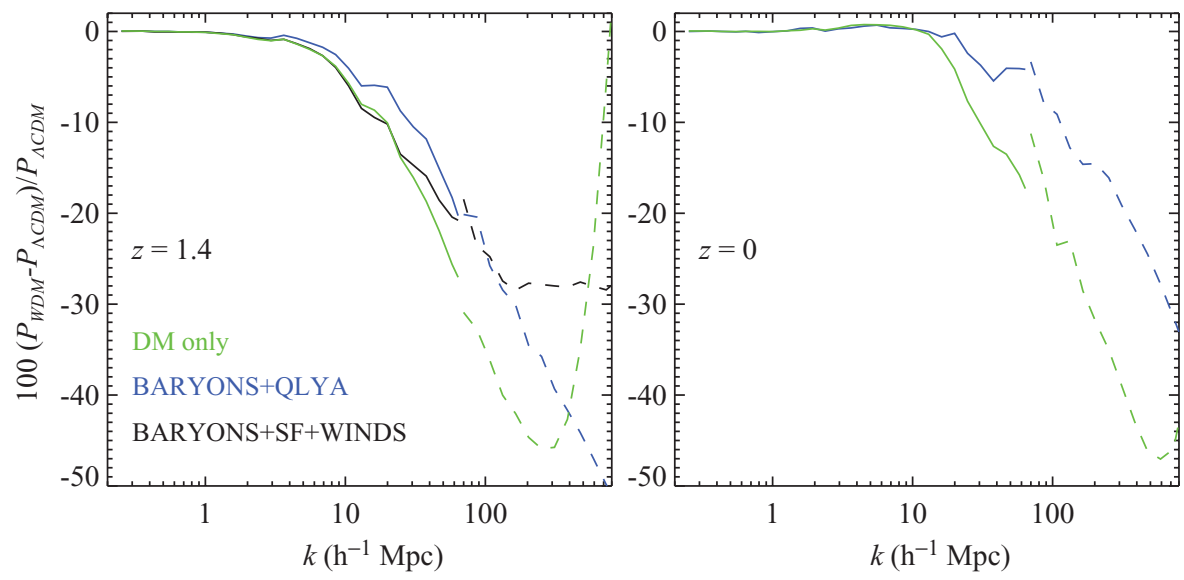

Figure 5. WDM suppression for three different simulations with and without baryons. These three different simulations are compared with the corresponding $\Lambda \mathrm{CDM}$ run with the same initial conditions. DMONLY is the resulting percentage difference between the WDM and CDM non-linear matter power spectrum (green), BARYONS+QLYA includes cooling due to $\mathrm{H}$ and $\mathrm{He}$ (blue), and BARYONS+SF+WINDS, which includes star formation and strong galactic winds (black). The prescription used for the star formation processes is labelled as 'quick Ly $\alpha$ ' from Viel et al. (2004). We show two different redshifts: $z=1.4$ and $z=0$.

suppressed is of the order of $k \sim 1 \mathrm{hMpc}^{-1}$ for $1 \mathrm{keV}$ WDM particles. The authors found a fitting function that can be used to calculate the non-linear matter power spectrum in the WDM scenario from the $\operatorname{CDM} P^{\mathrm{nl}}(k)$ analogously to Equation (7):

$$
\mathcal{T}_{\text {nl }}^{2}(k) \equiv P_{\mathrm{wdm}}(k) / P_{\mathrm{cdm}}(k)=\left(1+(\alpha k)^{v l}\right)^{-s / v},
$$

where

$$
\alpha\left(m_{\mathrm{wdm}}, z\right)=0.0476\left(\frac{1 \mathrm{keV}}{m_{\mathrm{wdm}}}\right)^{1.85}\left(\frac{1+z}{2}\right)^{1.3},
$$

and $v=3, l=0.6$, and $s=0.4$ are the fitting parameters. This function is applied by first calculating the non-linear matter power spectrum using $\Lambda \mathrm{CDM}$ parameter (e.g. from $\mathrm{CAMB}$ ) and then multiplying by the square of the WDM 'transfer function'.

Assuming WDM to be thermal relic fermions, their relic velocities have a Fermi-Dirac distribution, which can be added to the proper velocities calculated from the gravitational potentials from linear theory. The velocities for some of the WDM models they use can be found to be: $v_{\text {th }} \in\{27.9,11.5,4.4,1.7,0.7\} \mathrm{km} \mathrm{s}^{-1}$ for $m_{\mathrm{wdm}} \in$ $\{0.25,0.5,1,2,4\} \mathrm{keV}$, respectively. For comparison, the typical root mean square (r.m.s.) value for the velocity in a $\Lambda \mathrm{CDM}$ run is $v_{\mathrm{g}} \sim 30 \mathrm{~km} \mathrm{~s}^{-1}$, so it is significantly larger than any thermal velocities of WDM particles in the models that are still allowed by for example the Ly $\alpha$ forest $\left(m_{\mathrm{wdm}} \gtrsim 2 \mathrm{keV}\right)$.

It has been shown by many authors (e.g. Schaye et al. 2010; van Daalen et al. 2011; Casarini et al. 2011; Semboloni et al. 2011) that baryons, making up 17\% of the total matter density, affect the distribution of DM on small scales significantly. Simple hydro-dynamical simulations in WDM and $\Lambda$ CDM were run by Viel et al. (2012). They include a prescription for radiative cooling and heating, where all the cooling comes from Hydrogen and Helium (Katz et al. 1996 , as in) and no metal cooling is considered. The prescription for modelling the cooling and the star formation criterion are described in more detail in Viel, Haehnelt, \& Springel (2004) and is called 'quick Ly $\alpha$ ', since it can be used in order to speed up the hydro-dynamics with practically no impact on the Ly $\alpha$ forest flux statistics (this simulation is labelled as 'BARYONS+QLYA'). A further simulation has also been run that uses a more refined star formation criterion and strong galactic winds powered by the thermal feedback of supernovae (this simulation is labelled as 'BARYONS+SF+WINDS').

We plot in Figure 5 the resulting percentage difference between a $1 \mathrm{keV}$ WDM and CDM non-linear matter power spectrum, where both come from simulations that include cooling and heating processes from the ultraviolet background and a simple star formation criterion. Out of these simulations, one included galactic winds had to be stopped at $z=1.2$ due to limited computational resources and is therefore plotted above this redshift. It can be seen in Figure 5 that the inclusion of baryonic processes can have a very significant scale and redshift-dependent effect on the suppression signal of WDM. It seems likely that some baryonic processes become more efficient in a collapsing overdensity field that has been smoothed. Because the baryonic processes affect the power on small scales, this can erase the suppression from WDM, which is relevant on similar scales (see also Gao \& Theuns 2007).

It has also recently been reported by the authors of the OWLS simulations (van Daalen et al. 2011; Semboloni et al. 2011 , etc.) that the effects of baryonic processes, in particular the feedback from active galactic nuclei (AGN), can become dominant on scales that are significant to cosmology. This is 
certainly an important issue to consider in the future in order to realistically model the non-linear matter power.

\subsubsection{The WDM halo model}

It is interesting to note that even in the standard CDM scenario with WIMPy DM particles there exists a minimum freestreaming halo mass, which is very low. Green, Hofmann \& Schwarz (2005); Hofmann, Schwarz \& Stöcker (2001); Schneider et al. (2013b) find such CDM minimum haloes have masses of $M \sim 10^{-6} M_{\odot}$. In WDM models, this minimum mass is significantly larger. We explore this and other side effects of the primordial free-streaming of WDM on the properties and distribution of DM haloes in this section with reference to mostly the work of Markovič et al. (2011); Smith \& Markovič (2011); Schneider et al. (2012) and less so that of Cooray, $\mathrm{Hu} \&$ Miralda-Escude (2000); Cooray \& Sheth (2002); Abazajian et al. (2005); Zavala et al. (2009); Dunstan et al. (2011); Lovell et al. (2012); Pacucci et al. (2013). We do not discuss the work of Angulo et al. (2013); Schneider et al. (2013b) here in detail, but it is worth noting that they also modified the halo mass function such that it works well in fitting the results of numerical methods.

The halo model of large-scale structure offers a tool to quantify the non-linear structure growth. It is based on the spherical collapse model, where the over-densities of the matter density field collapse as spherically symmetric objects. In the most rudimentary form, the halo model assumes that all matter can be found within DM haloes, which merge into larger and larger haloes with time (i.e. 'bottom-up'), stopping only around the present time, when further non-linear collapse is halted by the emergence of the 'dark energy' component ${ }^{7}$.

The halo model assumes that halo positions are sampled from the linear theory matter distribution. As a result, there are two main contributions to the non-linear matter power spectrum. Firstly, the two-halo term, $P^{\mathrm{C}}(k)$, which dominates on large scales, encodes the correlation between different haloes and is equal to the linear matter power spectrum on large scales, $P^{\text {lin }}$. Secondly, the one-halo term, $P^{\mathrm{P}}(k)$, refers to the correlations within a halo and therefore depends mostly on the Fourier transform of the density profile of the halo, $\tilde{\rho}(k, M, z)$. Both terms depend on the number of haloes as a function of halo mass, $d n / d M$, which can be found to a reasonable approximation using analytic arguments or more usually measured from numerical simulations. The total nonlinear matter power spectrum from the halo model is a sum of the two terms:

$$
\begin{gathered}
P_{\mathrm{nl}}^{\mathrm{P}}(k, z)=\frac{1}{(2 \pi)^{3}} \int d M \frac{d n}{d M}\left[\frac{\tilde{\rho}(k, M, z)}{\rho_{\mathrm{m}, 0}}\right]^{2}, \\
P_{\mathrm{nl}}^{\mathrm{C}}(k, z)=P^{\operatorname{lin}}(k, z)\left[\int d M \frac{d n}{d M} b(M, z) \frac{\tilde{\rho}(k, M, z)}{\rho_{\mathrm{m}, 0}}\right]^{2} .
\end{gathered}
$$

\footnotetext{
${ }^{7}$ In $\Lambda \mathrm{CDM}$, this would have happened $\sim 5 \times 10^{9}$ years ago.
}

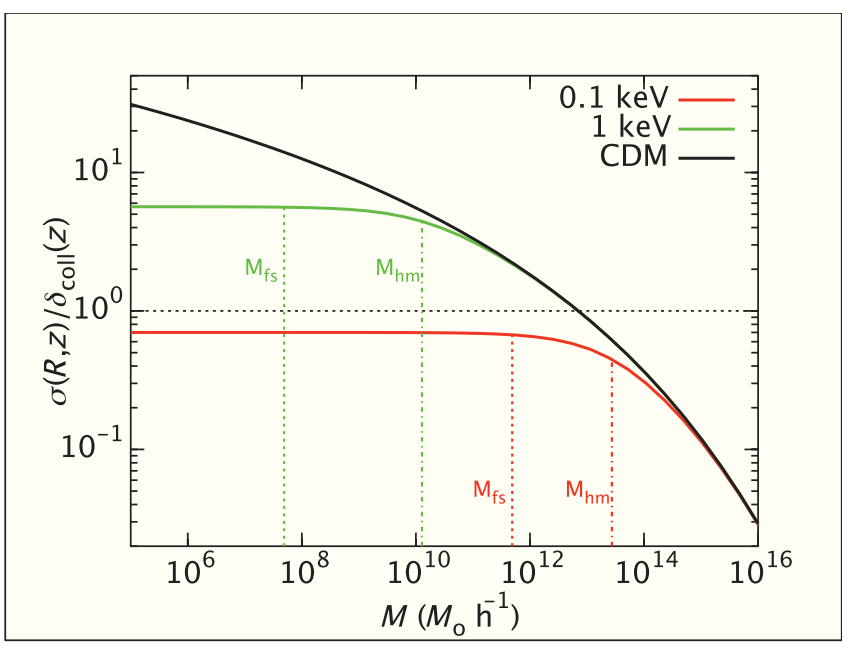

Figure 6. The root-mean-square density fluctuation for CDM (top, solid line), $0.1 \mathrm{keV}$ WDM (bottom), and $0.25 \mathrm{keV}$ (middle). The $\sigma(M)$ flattens off for the smallest halo masses in the WDM model, as one would expect for any smoothed field. The dotted black line indicates the critical over-density for spherical collapse.

Attempts have been made by Smith \& Markovič (2011); Schneider et al. (2012); Dunstan et al. (2011) to extend the halo model to WDM scenarios by modifying its ingredients. They use the WDM linear power spectrum to calculate a new mass function using the Sheth \& Tormen (1999) prescription. They make the conservative assumption that the halo profiles are unchanged relative to CDM. It is in the one-halo term of the power spectrum that the effects of free-streaming of WDM are seen most strongly. This is because of the difference in the r.m.s. fluctuation, $\sigma(R)$, which becomes suppressed at small $R$ in a WDM universe. This effect is shown in Figure 6 for two rather extreme WDM models, with very low particle masses. We plot this to show that for very low-mass DM particles (this is effectively HDM), the over-density field variance never reaches the necessary value for spherical collapse. This results in an extreme suppression of the formation of structure, ruling out the domination of the DM density by HDM.

We explore the effect of WDM on the mass functions in Figure 7. As expected, the number density for the smallest haloes is reduced in the case of WDM. This is shown most visibly in the left panel of Figure 7. This is useful for comparison to the general assumption of the absence of haloes below the free-streaming halo mass, defined in Equation (11) (Avila-Reese et al. 2001). The definition of the free-streaming mass is somewhat arbitrary, because it does not really correspond to a physical halo, since it does not include the density contrast parameter, $\Delta$ (as it does in Sommer-Larsen \& Dolgov 2001). For this reason Schneider et al. (2012) proposed to define instead the half-mode halo mass, which denotes the halo mass at which the mass functions become suppressed 

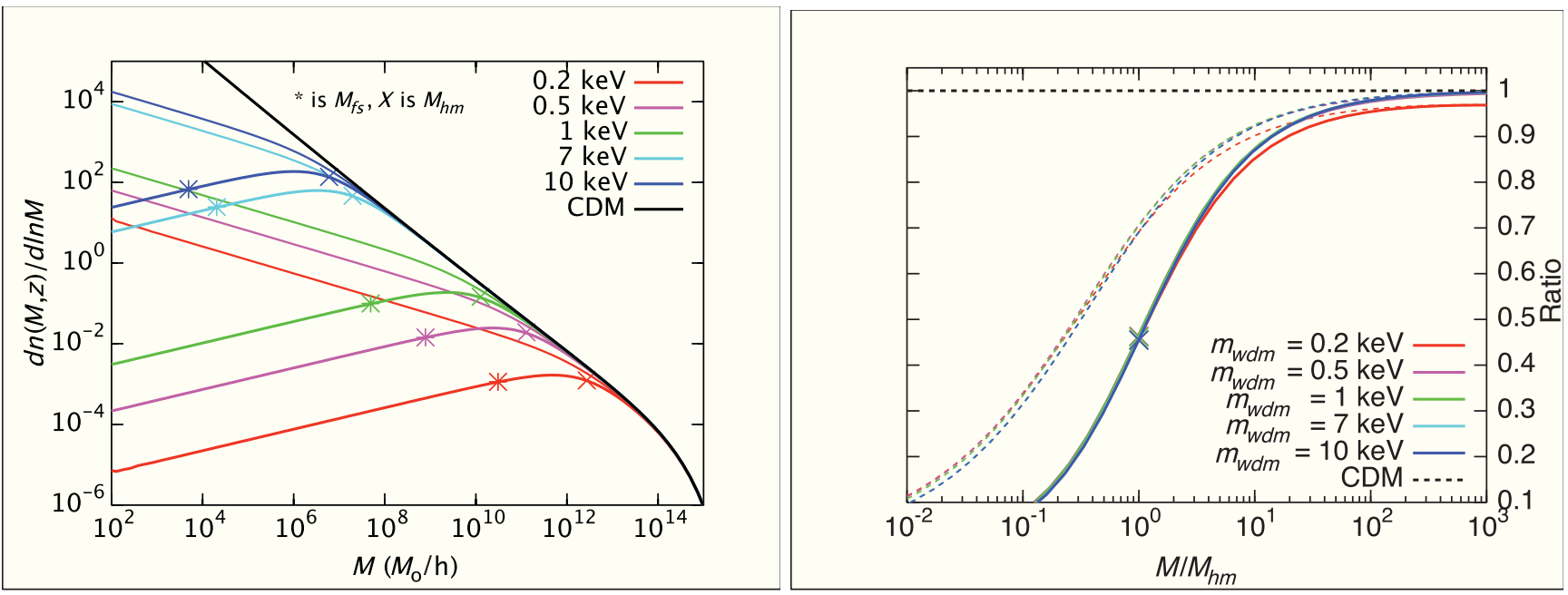

Figure 7. On the left we plot the mass functions from Sheth \& Tormen (1999) (dotted) vs. Schneider et al. (2012) fit to simulations (solid). On the right we show the re-scaled ratios of these mass functions by the half-mode halo mass, $M_{\mathrm{hm}}$. The half-mode and the free-streaming halo masses are plotted with crosses and asterisks, respectively.

by a factor of $1 / 2$ :

$$
\begin{aligned}
\lambda_{\mathrm{hm}} & =2 \pi \alpha\left(2^{v / 5}-1\right) \quad \text { and } \\
M_{\mathrm{hm}} & =\frac{4 \pi \bar{\rho}}{3}\left(\frac{\lambda_{\mathrm{hm}}}{2}\right)^{3},
\end{aligned}
$$

where $\alpha$ comes from Equation (8).

Schneider et al. (2012) examined the halo model in comparison to N-body simulations. They re-scaled the halo masses with respect to the half-mode mass, $M_{\mathrm{hm}} \approx 2.7 \times$ $10^{3} M_{\mathrm{fs}}$, rather than the free-streaming mass as above. They find the simple fitting formula:

$$
\frac{d \tilde{n}_{\mathrm{wdm}}}{d n_{\mathrm{wdm}}}=\left(1+\frac{M}{M_{\mathrm{hm}}}\right)^{-\alpha}
$$

to match their simulation results well without the need to apply an artificial step function. The single fitting parameter, $\alpha=0.6$, was able to match the simulations with less than 5\% r.m.s. error. Dunstan et al. (2011) find very similar results. In Figure 8, we plot the eight-times free-streaming mass against the WDM particle mass, $m_{\mathrm{wdm}}$, as well as the half-mode mass.

This modification was motivated by the simulated mass function declining much more steeply than the Sheth \& Tormen (1999) already seen by Zavala et al. (2009). This suggests that Sheth \& Tormen (1999) prescription underestimates the effect of WDM on the mass functions. On the other hand, there could be unforeseen resolution effects coming from the simulations. However, this is unlikely since there are usually spurious haloes created in WDM simulations, which for the Zavala et al. (2009) simulations increases the mass function $M \lesssim 10^{9} M_{\odot}$.

Interestingly, Schneider et al. (2012) also suggest a rescaling of the concentration parameter to suit the simulation results better. We also plot the NFW (Navarro et al. 1997)

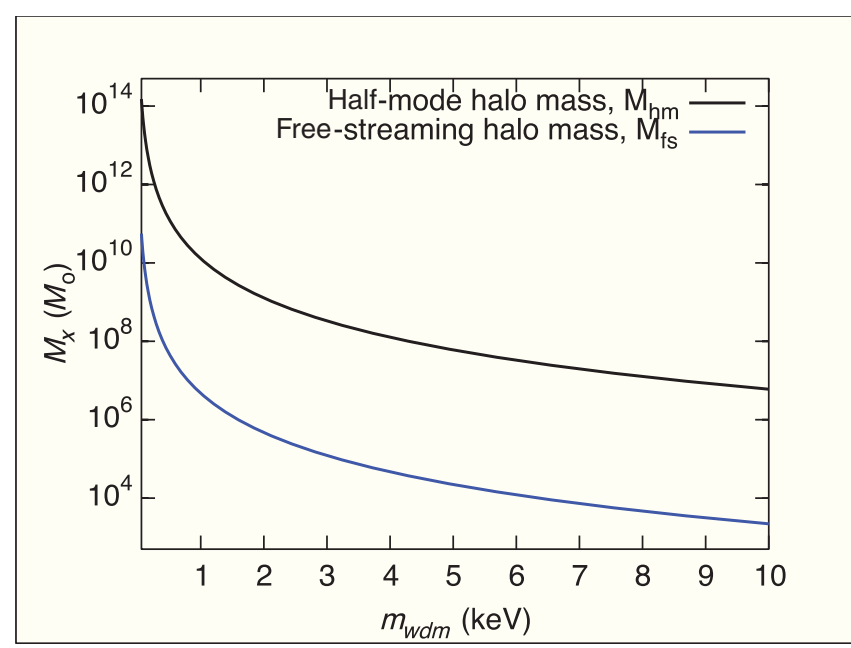

Figure 8. This figure compares the halo mass that corresponds to the ratio between WDM and CDM mass functions falling to a half, called the halfmode mass, $M_{\mathrm{hm}}$, and the free-streaming halo mass for WDM particles with masses $m_{\mathrm{wdm}}, M_{\mathrm{fs}}$.

halo density profiles calculated using the new WDM concentration parameter (Seljak 2000) re-scaling in Figure 9.

Unfortunately, the modifications to the halo model do not seem to adequately describe the evolution of the WDM suppression with redshift. For this reason it is still the Viel et al. (2012) fitting function for the $P_{\mathrm{wdm}}^{\mathrm{nl}}(k)$ that best fits the results from the above-mentioned simulations. We plot the ratios of the WDM vs. CDM non-linear matter power spectra in Figure 10.

Very recently however, Benson et al. (2012); Schneider et al. (2013b) proposed that the mass functions should be calculated with the standard Sheth \& Tormen (1999) prescription, but using a sharp-k filter to find the $\sigma(R)$ instead of the real-space top-hat. This seems to describe the redshift 

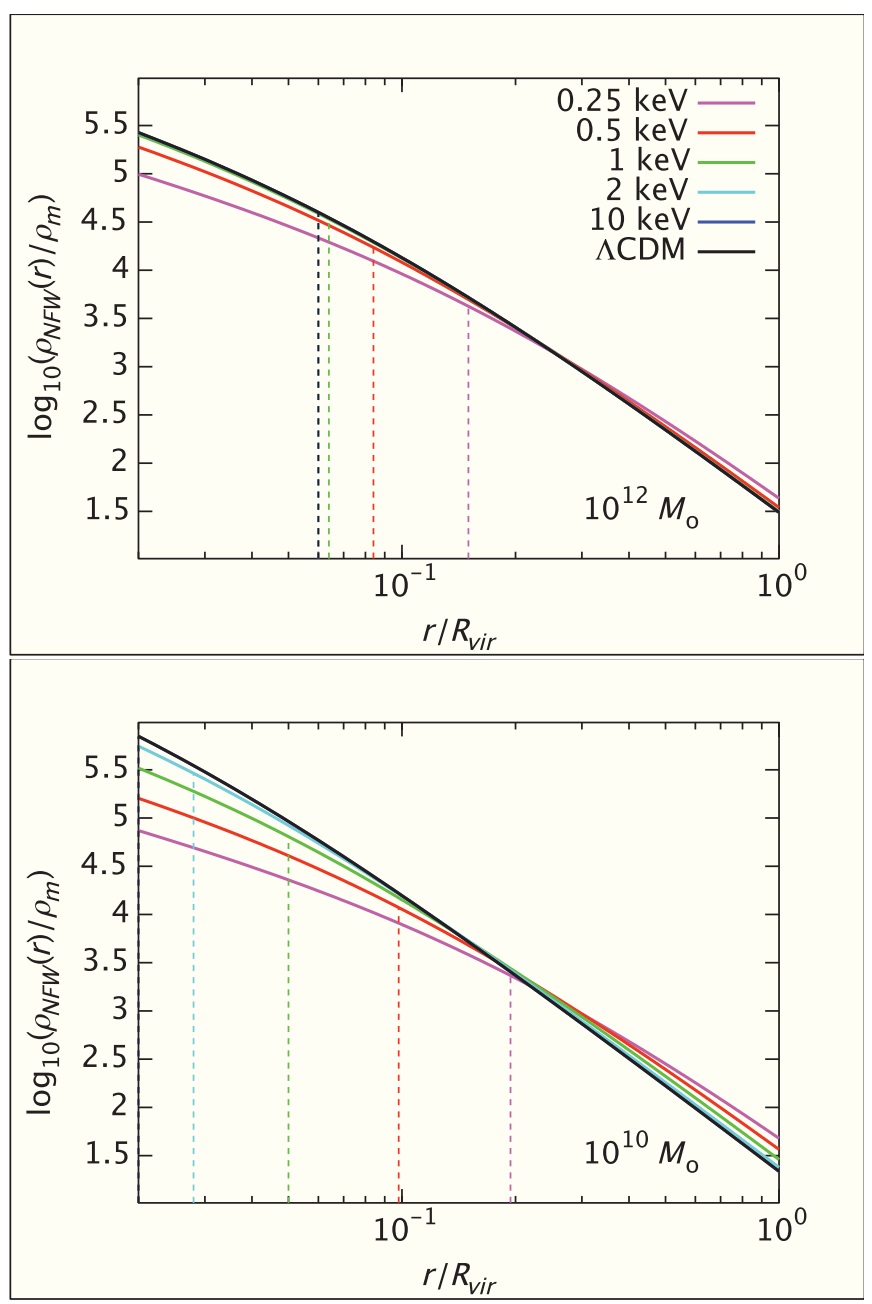

Figure 9. NFW (Navarro et al. 1997) halo density profiles for two different halo masses $\left(10^{10}\right.$ and $\left.10^{12} M_{\odot}\right)$ calculated with the re-scaled concentration parameter from Schneider et al. (2012) for WDM models with $m_{\mathrm{wdm}} \in$ $\{0.25,0.5,1.0,2.0,10.0\} \mathrm{keV}$. The dashed vertical lines correspond to the free-streaming lengths in the different WDM models, re-scaled by the virial radius of the halo.

evolution of the WDM suppression better. On the other hand, Pacucci et al. (2013) propose to raise the collapse threshold to emulate the difficulty of collapse in the WDM scenario and calculate the mass functions at high redshift.

\section{PRESENT AND FUTURE CONSTRAINTS}

Using the methods described above, one can model the structure in the universe and compare the resulting power spectra to observations. In this section we will review the constraints that are obtained and could be obtained from the two most powerful small-scale observables: the Ly $\alpha$ forest and weak lensing.

\subsection{The Lyman- $\alpha$ forest}

The Ly $\alpha$ forest, the absorption induced by intervening neutral hydrogen along the line-of-sight to a distant quasar, is a powerful cosmological tool ideally suited to probe the clustering of matter over a range of scales from below the Mpc to hundreds of Mpc and from $z=2$ up to $z=6$ (for a review see Meiksin 2009). The idea behind the cosmological applications of the Ly $\alpha$ forest is to relate flux fluctuations,

$$
\delta_{\mathrm{F}}=\frac{F-\langle F\rangle}{\langle F\rangle},
$$

to matter fluctuations. This can be done in several ways and the simplest is to make use of the so-called Fluctuating GunnPeterson approximation ${ }^{8}$

$$
\tau_{\mathrm{GP}}=\frac{\pi e^{2}}{m_{e} c} f_{\alpha} \lambda_{\alpha} H^{-1}(z) n_{\mathrm{HI}},
$$

with $n_{\mathrm{HI}}$ being the neutral hydrogen density that relates the optical depth to the underlying density of neutral hydrogen, $f_{\alpha}$ being the oscillator strength, and $\lambda_{\alpha}=1215.67 \AA$ being the Ly $\alpha$ absorption wavelength. The assumption that the gas producing the absorption is in photoionisation equilibrium implies that $n_{\mathrm{HI}} \propto \rho^{2} T^{-0.7} / \Gamma$, where $\Gamma$ is the photoionisation rate. Furthermore, if one assumes that the gas temperature scales as $T=T_{0}(\rho /\langle\rho\rangle)^{\gamma}$, which is set by the balance between photo-heating and adiabatic cooling due to the expansion of the universe and has been found to be a good approximation of the gas thermal state at low-densities, one obtains (see Viel et al. 2002):

$$
\tau \propto A(z)\left(\frac{\rho}{\langle\rho\rangle}\right)^{\beta} \quad \text { with } \quad \beta=2-0.7 \gamma,
$$

where the redshift dependent $A$ factor will depend also on cosmological parameters, atomic physics, and on the photoionisation rate. The observed quantity is the transmitted flux $F=\exp (-\tau)$ and at first order it can be easily seen that flux fluctuations are related to the linear density contrast as $\delta_{\mathrm{F}} \propto-A \beta \delta_{\text {lin }}$. Non-linearities in the density fields and those induced by peculiar velocities complicate the picture above and simple analytical insights or semi-analytical models (Bi \& Davidsen 1997) must be replaced by more reliable and accurate hydrodynamic simulations of intergalactic medium structures performed either with smoothed-particle hydrodynamics, Eulerian, or adaptive mesh refinements codes.

The use of Ly $\alpha$ forest data to probe matter clustering has been pioneered by Croft and co-workers at the end of the 90s: a measurement of the linear matter power spectrum at small scales and high redshift has been presented in Croft et al. (2002), by using high and medium resolution quasar spectra together with the so-called 'effective bias' method, $P_{\mathrm{F}}(k)=b^{2}{ }_{\text {eff }}(k, z) \times P_{\text {lin }}(k)$, that allowed an inversion of the one-dimensional (1D) flux power to infer the underlying matter power spectrum. After that, Viel et al. (2004) used a set of about 30 high-resolution high signal-to-noise quasar spectra taken with the UVES/VLT spectrograph and

\footnotetext{
${ }^{8}$ This approximation neglects non-linearities induced by the fact that the signal is in redshift space.
} 

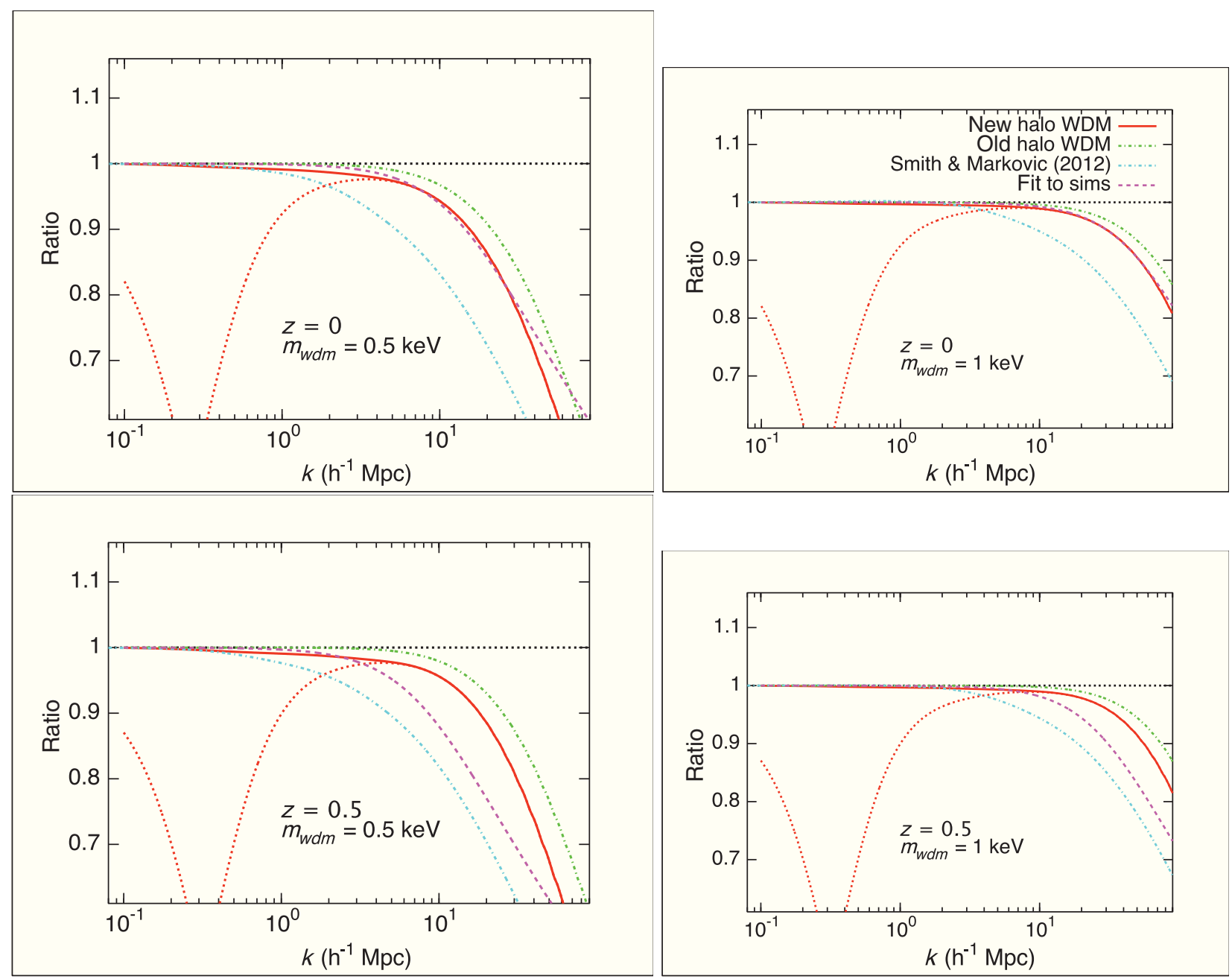

Figure 10. Ratios of non-linear matter power spectra in the different models, $P_{\mathrm{wdm}}^{\mathrm{nl}}(k) / P_{\mathrm{cdm}}^{\mathrm{nl}}(k)$ for $500 \mathrm{eV}$ and $1 \mathrm{keV}$ WDM particles at $z=0$ on top and $z=0.5$ at the bottom. The dotted red lines show the $P^{\mathrm{C}}(k)$ and $P^{\mathrm{P}}(k)$ dominating at small and large $k$, respectively. The red solid lines show the halo model modified like in Schneider et al. (2012). The cyan dash-dotted line shows the older modification of the halo model by Smith \& Markovič (2011). The green dash-dotted line is the simplest version of the halo model with standard Sheth \& Tormen (1999) mass functions.

a suite of full hydrodynamic simulation, that explored several thermal histories, to derive the matter clustering at $z \sim$ 2.1 and $z \sim 2.7$. These data have been combined in a series of paper with WMAP data in order to probe the long-lever arm of the matter power spectrum and get constraints on the running of the spectral index and inflationary parameters (Viel, Haehnelt \& Lewis 2006). A new era in the Ly $\alpha$ forest field has entered with the advent of the SDSS survey that has allowed to obtain the 1D flux power spectrum from a set of 3000 low-resolution quasars in the range $z=$ $2.2-4.2$ over two decades of wavenumbers (McDonald et al. 2005) and to infer the linear matter power spectrum amplitude, slope, and curvature at $z=3$ and at a comoving scale of about $\sim 8 \mathrm{Mpc} \mathrm{h}^{-1}$ with unprecedented precision (McDonald et al. 2005), by means of approximate hydro simulations. Again the SDSS data have been combined with other large-scale structure probes to get very tight constraints in terms of neutrino mass fractions and cosmological param- eters like running of the spectral index and inflation (Seljak et al. 2006b). More recently, BOSS/SDSS-III has measured the three-dimensional (3D) clustering of the flux by exploiting the signal in the transverse direction from a set of 50000 quasar spectra: this new data set has allowed to measure at high significance the presence of baryonic acoustic oscillations (BAO) at $z \sim 2.2$ (Busca et al. 2013; Slosar et al. 2013) and a new measurement of the 1D flux power has also been recently provided (Palanque-Delabrouille et al. 2013).

Ly $\alpha$ forest data are currently providing the tightest constraints in terms of WDM properties and there are two main reasons for this. First of all, the 1D power spectrum is a projection of the 3D one and contains information down to very small scales,

$$
P_{1 \mathrm{D}, \mathrm{F}}=\frac{1}{2 \pi} \int_{k}^{\infty} P_{3 \mathrm{D}, \mathrm{F}}(y) y d y,
$$




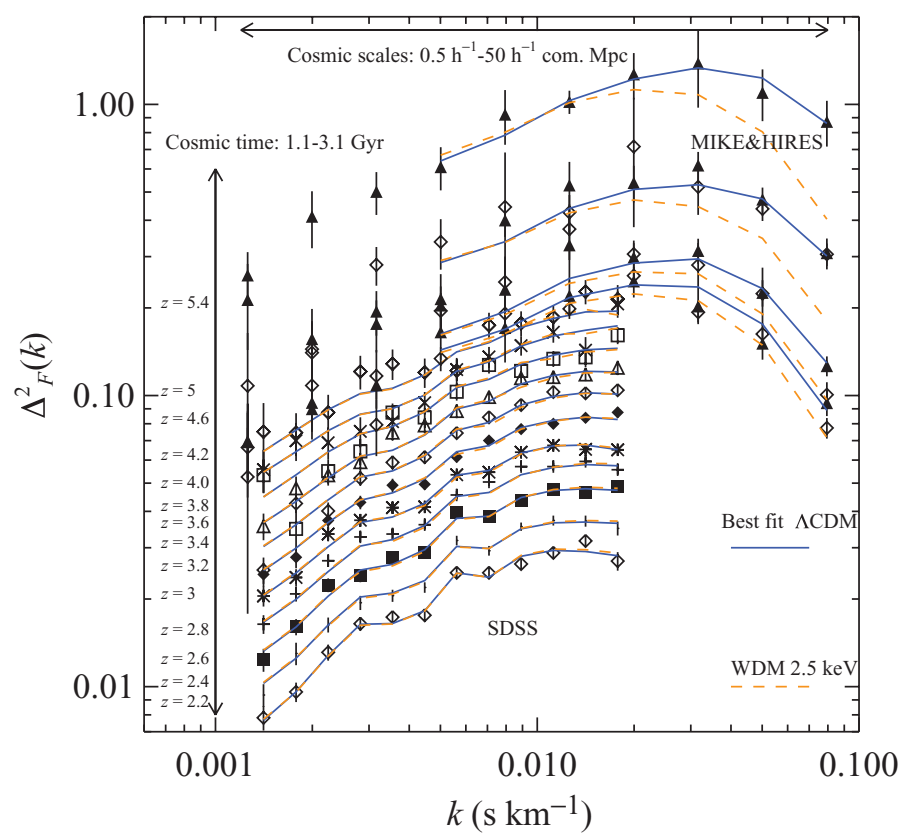

Figure 11. One-dimensional flux power spectrum in dimensionless units $\left(\Delta^{2}(k)\right.$ $\left.=P_{\mathrm{F}}(k) \times k /(2 \pi)\right)$ for the SDSS (McDonald et al. 2005) and MIKE+HIRES (Viel et al. 2013a) data sets. These data points $\operatorname{span} z=2.2-5.4$, a period of about 2 Gyrs and about two decades in wavenumber space. The best fit $\Lambda \mathrm{CDM}$ model is shown as the blue line, while the orange dashed curves are for a WDM model with a $m_{\mathrm{wdm}}=2.5 \mathrm{keV}$, which is excluded by the data at very high significance (note that in this case the other parameters have been kept to their best fit values and only $m_{\mathrm{wdm}}$ is changed).

and thereby is sensitive to the cut-off induced by WDM. Secondly, Ly $\alpha$ forest data span high redshift where the WDM cut-off in terms of matter power is more pronounced and much closer to the linear behaviour (see Section 2); in fact the $\mathrm{Ly} \alpha$ forest flux power is particularly sensitive at environments around the mean density, closer to the linear regime, and this is especially true at high redshift, due to the strong evolution of the mean flux level. There is also another reason that plays an important albeit minor role: the thermal broadening depends on the temperature, which becomes colder at high redshift, and is a fixed number in velocity space while the free-streaming length scales as $\sqrt{1+z}$, making the thermal contribution to a possible WDM cut-off less prominent at high redshift.

In Table 1 we present a summary of the constraints, in terms of the mass of a thermal relic, that have been obtained by using $\operatorname{Ly} \alpha$ forest data.

The first constraint was obtained by Narayanan et al. (2000): by using N-body simulations only and a set of eight high-resolution spectra, the authors looked also at the flux probability distribution function and not just at the flux power and obtained a lower limit of $0.75 \mathrm{keV}$. The main limitations of this work were due to the fact that no hydro simulations were used and a proper marginalisation over nuisance parameters was not done. Viel et al. (2005) used instead the effective bias method of Croft et al. (2002) and a set of full hydro simulations to explore the bias in WDM scenarios us- ing high-resolution UVES spectra at $z=2.1,2.7$. In this case, the authors found a $2 \sigma$ lower limit of $0.55 \mathrm{keV}$ for a thermal relic and the nuisance parameters were accounted for (and marginalised over) by allowing an extra normalisation error on the data. In this paper, the authors also quote a $2 \sigma$ lower limit of $2 \mathrm{keV}$ for a sterile neutrino in the so-called DodelsonWidrow scenario (Dodelson \& Widrow 1994) and an upper limit for a gravitino of $16 \mathrm{eV} 2 \sigma$ C.L. in a model for which this particle is not the total amount of DM. Subsequently, Seljak et al. (2006a) exploited the unique capabilities of the SDSS flux power spectrum of McDonald et al. (2005) (about 3000 low-resolution low signal-to-noise QSO spectra spanning the redshift range $z=2.2-4.2$ ) and showed that the constraints derived from this data set were much tighter due to the wide redshift range probed that allowed to break the degeneracies between cosmological and thermal/nuisance parameters. They obtained a limit of $m_{\mathrm{wdm}}>2.5(14) \mathrm{keV}$ for a thermal relic (sterile neutrino) at the $2 \sigma$ C.L. The analysis made was based on a set of approximate hydro simulations that however explored fully the multi-dimensional likelihood space. The numbers derived above found confirmation in an independent analysis of the SDSS data made by Viel et al. (2006) in which a suite of full hydro-dynamical simulations were used at the expenses of a relative poor scanning of the multi-dimensional likelihood space obtained with a Taylor expansion of the flux power. In this work, the limits found were: $m_{\mathrm{wdm}}>2(10) \mathrm{keV}$ for a thermal relic (sterile neutrino) 
Table 1. Summary of the constraints obtained on the mass of a WDM relic by using Ly $\alpha$ forest data. Apart from Narayanan et al. (2000) all the other quoted values are $2 \sigma$ confidence level obtained in a Bayesian analysis.

\begin{tabular}{lcll}
\hline \hline Reference & $m_{\text {wdm }}(\mathrm{keV})$ & \multicolumn{1}{c}{ Data } & \multicolumn{1}{c}{ Notes } \\
\hline Narayanan et al. (2000) & $>0.75$ & \multicolumn{1}{c}{8 high-res. } & Not marginalised, N-body only \\
Viel et al. (2005) & $>0.55$ & 30 UVES spectra & Eff. bias, hydro sims. \\
Seljak, Slosar \& McDonald (2006b) & $>2.5$ & $\sim 3000$ SDSS spectra & Approx. hydrod., full likelihood expl. \\
Viel et al. (2006) & $>2$ & $\sim 3000$ SDSS spectra & Full hydro, approx. likelihood expl. \\
Viel et al. (2008) & $>4.5$ & $\sim 3000$ SDSS, $\sim 60$ Keck sp. & Full hydro, approx. likelihood expl. \\
Viel et al. (2013a) & $>3.3$ & 28 high- $z$ MIKE + HIRES sp. & Full hydro, good likelihood expl. \\
\hline \hline
\end{tabular}

at the $2 \sigma$ C.L., in good agreement with the analysis of Seljak et al. (2006a).

After this Viel et al. (2008), explored the very high redshift regime by using 55 high-resolution Keck spectra at $z=$ $2-6.4$ and obtained the limits $m_{\mathrm{wdm}}>1.2(5.6) \mathrm{keV}$ for a thermal relic (sterile neutrino) at the $2 \sigma$ C.L. However, these limits greatly improved by adding the SDSS data that allowed to break the degeneracies between thermal and WDM cut-offs. A colder (hotter) IGM will result in an increase (suppression) of the flux power due to the thermal broadening of the lines, which is different: this 'thermal' effect could either erase or boost the WDM-induced suppression. The advantage of having a wide redshift range allows to appreciate the different redshift evolution of the WDM and thermal cut-offs and to lift or break their mutual degeneracies. Thanks to their constraining power, these data allowed to obtain $m_{\mathrm{wdm}}>4.5(28) \mathrm{keV}$ for a thermal relic (sterile neutrino) at the $2 \sigma$ C.L. In this analysis a second-order Taylor expansion of the flux was used, but again the parameter space was not explored fully and large numerical corrections were made to the flux power in the highest redshift bins.

In Viel et al. (2013a) these numbers have been revised by using a very comprehensive grid of hydro simulations that embrace a conservative range of different thermal history. At these high redshift it is also likely that galactic feedback and astrophysical effects have a much weaker impact in terms of flux power (Viel, Schaye \& Booth 2013b). In this case the marginalisation over nuisance parameters has been made fully in the most relevant parameter space and hydro simulations at higher resolutions have been employed. The data used were the highest redshift Keck spectra complemented by an equal number of MIKE (Magellan spectrograph) at poorer resolution. The flux power spectrum has been measured at $z=4.2,4.6,5,5.4$ down to the scales of $k \sim 0.1 \mathrm{~s}$ $\mathrm{km}^{-1}$, roughly corresponding to (very non-linear) scales $\lambda=$ $50 \mathrm{~h}^{-1} \mathrm{Mpc}$. The final results, that also allow for a conservative extra error on the data side of about $30 \%$ and is not sensitive to continuum fitting uncertainties, give $m_{\mathrm{wdm}}>3.3 \mathrm{keV}$ for a thermal relic at the $2 \sigma$ C.L., after having marginalised over nuisance, ultra-violet fluctuations, instrumental resolution, and noise of the spectrograph. From this data set and analysis the authors concluded that thermal relics of masses $1 \mathrm{keV}, 2 \mathrm{keV}$, and $2.5 \mathrm{keV}$ are disfavoured by the data at about the $9 \sigma, 4 \sigma$, and $3 \sigma$ C.L., respectively (see Figure 11).
WDM models where there is a suppression in the linear matter power spectrum at (non-linear) scales corresponding to $k=10 \mathrm{hMpc}^{-1}$, which deviates more than $10 \%$ from a $\Lambda \mathrm{CDM}$ model, are disfavoured by the data. Given this limit, the corresponding 'free-streaming mass' below which the mass function may be suppressed is $\sim 2 \times 10^{8} h^{-1} \mathrm{M}_{\odot}$. There appears thus to be very little room for a contribution of the free-streaming of WDM to the solution of what has been termed the small-scale crisis of CDM.

These models have been refined further by accounting for the case of a mixed C+WDM model in Boyarsky et al. (2009a), where an analysis of the SDSS and UVES data was presented. In this work the main results were expressed in terms of a non-resonantly produced sterile neutrino and give $m_{\mathrm{NRP}}>8 \mathrm{keV}$ (frequentist $99.7 \%$ confidence limit) or $m_{\mathrm{NRP}}>12.1 \mathrm{keV}$ (Bayesian $95 \%$ credible interval) in a pure WDM model. For the mixed model, they obtained limits on the mass as a function of the WDM fraction (percentage) to be smaller than $60 \%$ for any value of the WDM particle mass (frequentist $99.7 \%$ confidence limit), while the Bayesian joint probability allows any value of the mass (for $m_{\mathrm{NRP}}>5 \mathrm{keV}$ ) at the $95 \%$ confidence level, provided that the fraction of WDM is below 35\%, for any value of the WDM particle mass. This limit can be roughly translated into a thermal relic mass and implies that fractions of WDM below 35\% can be accommodated only for masses above $m_{\mathrm{wdm}}>1.1 \mathrm{keV}$.

In Boyarsky et al. (2009b) a mechanism of resonantly produced sterile neutrino, that occurs in the framework of the $v$ MSM (the extension of the Standard Model with three right-handed neutrinos), is analysed. Here it was shown that their cosmological signature can be approximated by that of mixed $\mathrm{C}+\mathrm{WDM}$ and for each mass greater than or equal to $2 \mathrm{keV}$, there exists at least one model of sterile neutrino accounting for the totality of DM, and consistent with Ly $\alpha$ and other cosmological data. However, the transfer function for such candidates is quite different from the one of the thermal relic and no direct comparison with thermal masses can be made.

These lower limits seem to be conflicting with the upper limits obtained on the masses of such particles coming from the observations of the cosmic X-ray background and are: $m_{\text {sv }}<1.8 \mathrm{keV}$ at $95 \%$ confidence (Boyarsky et al. 2006). In fact, in addition to the dominant decay mode into three active neutrinos, the light sterile neutrino can decay into an 
active one and a photon with the energy $E_{\mathrm{s}}=m_{\mathrm{s}} / 2$. Thus, there exists a possibility of direct detection of neutrino decay emission line from the sources with big concentration of DM, e.g. from the galaxy clusters (Abazajian, Fuller \& Tucker 2001). Similarly, the signal from radiative sterile neutrino decays accumulated over the history of the universe could be seen as a feature in the diffuse extragalactic background light spectrum. However, the constraints above assume a very simple model for sterile neutrino production and can be circumvented by considering other models (Boyarsky et al. 2009a).

Overall, Ly $\alpha$ offers a unique probe of the matter power spectrum down to very small scales and the tightest constraints in terms of CDM coldness. The most recent constraint $m_{\mathrm{wdm}}>3.3 \mathrm{keV}$ is suggesting that the cosmic web as probed by the Ly $\alpha$ forest data is quite cold and the values of WDM masses $(0.5-1.5 \mathrm{keV})$ that are typically used in order to solve the missing satellite, the cusp-core, and the 'too-bigto-fail' problems for the dynamical properties of the most massive dwarf galaxies at low redshift are in strong tension with the limits above.

\subsection{Cosmic weak lensing}

In order to complement the $\operatorname{Ly} \alpha$ constraints on the thermalised DM particle mass, we could look at the cosmological data of sources seen at different redshifts (tomography). An example of such a probe is gravitational lensing, being also the only probe that does not rely on making assumptions about the coupling between dark and luminous objects in that it probes directly the total gravitational potential.

In particular, the weak gravitational lensing induced in the background distribution of distant galaxy images is known as cosmic shear and is only detectable statistically. Cosmic shear is the weak lensing signal that is induced by the $3 \mathrm{D}$ distribution of mass in the universe. We wish to describe in this section how to theoretically calculate the weak lensing angular power spectrum, given a 3D DM power spectrum found in the previous sections (see also Bartelmann \& Schneider 2001). We would like to consider theoretical weak lensing power spectra similar to those that could be obtained by future surveys like Euclid (Amendola et al. 2012; Refregier et al. 2010) and present the effect of small-scale WDM-induced suppression. This will be useful for making predictions for constraints and measurements as done in Markovič et al. (2011); Smith \& Markovič (2011); Viel et al. (2012).

An approximate shear power spectrum can be calculated from the halo model (Cooray et al. 2000) and is made up of two terms, analogously to Equation (15), the one-halo (or Poisson) term and the two-halo (or correlation) term where in order to project the matter power spectrum to two-dimension, the small angle (Limber 1953; LoVerde \& Afshordi 2008) approximation must be made.

Markovič et al. (2011); Smith \& Markovič (2011); Viel et al. (2012) considered how to measure the WDM particle mass using observations of cosmic shear power spectra.
From an observer's point of view, the image of each galaxy is distorted by gravitational lensing effects of all intervening matter. Therefore, the cosmic shear power spectra are closely related to the matter power spectrum integrated over redshift. Future surveys are expected to use broadband photometry to estimate the redshifts of the observed galaxies. This should allow shear power spectra to be calculated at different redshifts, and also allow cross power spectra between redshifts (see Csabai et al. 2003, for a review).

The above-mentioned halo model approach assumes all sources at the same redshift for simplicity; however, we can expand the calculations to have a source redshift distribution and divide the source galaxies into redshift-determined tomographic bins. We may consider a cosmic shear survey that has a number of galaxies per unit redshift (Smail, Ellis \& Fitchett 1994):

$$
n(z)=z^{\alpha} e^{-\left(z / z_{0}\right)^{\beta}}
$$

The lensing power spectra are related to the 3D non-linear matter power spectra via

$$
C_{i j}(l)=\int_{0}^{\chi_{\mathrm{H}}} d \chi_{\mathrm{l}} W_{i}\left(\chi_{\mathrm{l}}\right) W_{j}\left(\chi_{\mathrm{l}}\right) \chi_{\mathrm{l}}^{-2} P_{\mathrm{nl}}\left(k=\frac{l}{\chi_{\mathrm{l}}}, \chi_{\mathrm{l}}\right),
$$

where $\chi_{1}\left(z_{1}\right)$ is the comoving distance to the lens at redshift $z_{1}$ and $W_{i}$ is the lensing weight in the tomographic bin $i$ :

$$
W_{i}\left(z_{\mathrm{l}}\right)=\rho_{\mathrm{m}, 0} \int_{z_{1}}^{z_{\max }}\left[\frac{n_{i}\left(z_{\mathrm{s}}\right)}{\Sigma_{\text {crit }}\left(z_{\mathrm{l}}, z_{\mathrm{s}}\right)}\right] d z_{\mathrm{s}},
$$

where

$$
\Sigma_{\text {crit }}\left(z_{1}, z_{\mathrm{s}}\right)=\frac{c^{2}}{4 \pi G} \frac{\chi_{\mathrm{s}}}{\chi_{\mathrm{ls}} \chi_{1}} \frac{1}{\left(1+z_{1}\right)},
$$

and the subscripts $s, 1$, and ls denote the distance to the source, the distance to the lens, and the distance between the lens and source, respectively.

In order to assess detectability of WDM by future weak lensing surveys, the works above calculate predicted error bars on the weak lensing power spectrum using the covariance matrix formalism (Takada \& Jain 2004) and assuming errors for a future realistic weak lensing survey with eight redshift bins in the range $z=0.5-2.0$ (see Figure 12). They additionally consider models of non-linear WDM structure to calculate the weak lensing power spectra. They find that for a survey like Euclid it seems to be sufficient to model the non-linearities using the HALOFIT prescription of Smith et al. (2003). The limits they predict for the WDM particle mass are at the same order of magnitude as those obtained from Ly $\alpha$ data Section 3.1 and therefore, they hold the potential to confirm the exclusion of $m_{\mathrm{wdm}} \lesssim 2 \mathrm{keV}$. In Table 2, we quote the actual predictions made. We note that a combination of Ly $\alpha$ and weak lensing can also be very promising in constraining the small-scale clustering of matter as done in Lesgourgues et al. (2007) for a standard CDM scenario. 

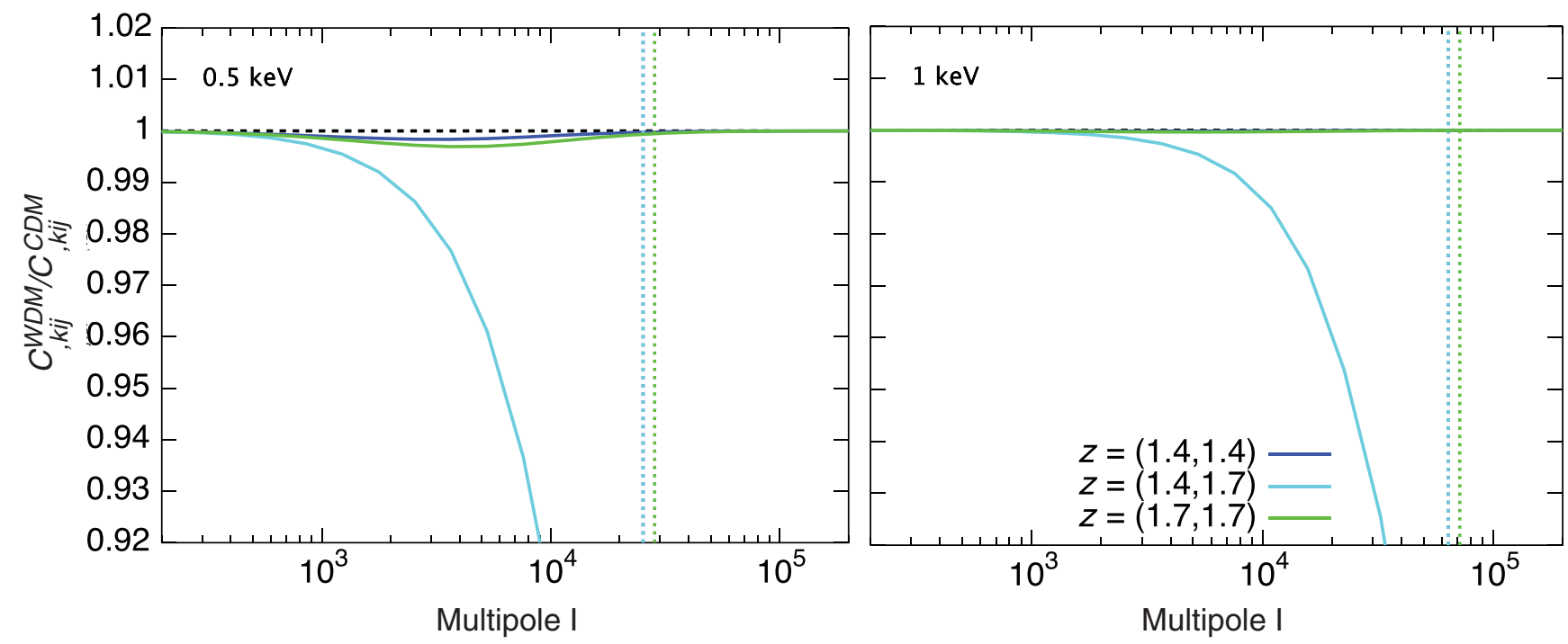

Figure 12. We plot the ratios between the weak lensing power spectra obtained from a Euclid-type survey. We plot the ratios of the cross-spectra of two tomographic bins, where we have used the Viel et al. (2012) fit for the WDM power spectrum from simulations for two different WDM models. The solid lines are the cross- and auto-correlation lensing power spectra. The dotted lines correspond approximately the $l_{\mathrm{fs}}$, i.e. the multipole value corresponding to the free-streaming scale, $k_{\mathrm{fs}}$, at the redshift or the bin (or the closer bin in the case of cross-spectra).

Table 2. Summary of the forecasts for the Euclid survey for constraining $m_{\mathrm{wdm}}$. The lower limits are quoted to $68 \%$ confidence; the fiducial model has been chosen as CDM (i.e. $\left.m_{\mathrm{wdm}} \rightarrow \infty\right)$.

\begin{tabular}{lclc}
\hline \hline Reference & $m_{\mathrm{wdm}}(\mathrm{keV})$ & \multicolumn{1}{c}{ Model } & Notes \\
\hline Markovič et al. (2011) & $>2.5$ & Unmodified HaLOFIT & Forecast: Euclid + Planck \\
Smith \& Markovič (2011) & $>2.6$ & Ad hoc WDM halo model & Forecast: Euclid + Planck \\
\hline \hline
\end{tabular}

\section{CONCLUSIONS}

This review has focussed on different approaches for modelling the non-linear structures in our universe in the $\Lambda \mathrm{WDM}$ model and on the possibilities of constraining such a scenario with two particular sets of data: Ly $\alpha$ forest and cosmic shear. We have made a simple choice for the WDM particle: a thermal relic with a $\sim \mathrm{keV}$ mass, a mass that had been proposed in the past to solve the so-called small-scale crisis of the standard $\Lambda \mathrm{CDM}$ cosmology.

We have decided to rely on a number of assumptions. Firstly, in the modelling of non-linear structure, we have neglected the contributions of baryonic processes (although they have been discussed) to the shape of the potential wells of haloes. We have also focussed more on the clustering of DM and less so on the profiles and substructures of individual DM objects.

Secondly, in types of observations we concentrated on Ly $\alpha$ forest and gravitational lensing and have not discussed for example other promising observables like the $21 \mathrm{~cm}$ line (see Sitwell et al. 2013) or the small-scale clustering of galaxies.

Thirdly, we drastically narrowed down the range of possible particle models of DM, allowing only for the particle mass (and therefore temperature) to vary, neglecting nonneutrino-like interactions and particle properties. We have made these choices in order to simplify the analysis. Any possibilities that were neglected here were omitted for reasons of practicality rather than plausibility or usefulness.

In summary, in order to describe a WDM regime, it is crucial to model the non-linearities in the matter power spectrum: thus, we have quoted the results of $\mathrm{N}$-body numerical simulations and described the modifications to the existing halo model. In particular, we have reported on simulations that resulted in a new WDM transfer function for the nonlinear power. This fitting function is useful for calculating theoretical WDM power spectra and comparing them to large-scale structure data. We have also touched upon the subject of baryonic physics, modelled by hydro-dynamical simulations. Even though such numerical prescriptions are yet uncertain, it is clear that before a measurement or constraint on the WDM mass is made, one must correctly model baryonic effects.

A possibility to disentangle cosmological and astrophysical effects and to break this degeneracy, could be to look at their potentially different redshift evolution. Whereas the WDM suppression increases with increasing redshift, the 
effect of baryons may have an entirely different signature. This would be important to model using numerical methods, but it would be a large undertaking as it would require extensive computational resources.

Another powerful tool for understanding and interpreting the large-scale structure is offered by the halo model. The halo model has now been modified and calibrated against $\mathrm{N}$-body simulations such that it is appropriate to use in the $\Lambda \mathrm{WDM}$ cosmology to predict the statistics of the large-scale structure. The new 'warm' halo model can be useful for a comparison with future galaxy surveys, where in order to compare the galaxy distribution measured from observations, one must populate the theoretical DM density field with galaxies. This WDM halo model has been developed with a rather physical motivation by Lesgourgues \& Tram (2011) constructing the density field from DM haloes that host galaxies. We have not discussed the clustering of galaxies explicitly, because competitive constraints should come only from small scales where the interpretation is not trivial and should rely on halo occupation distribution models. Therefore we have found it sufficient and more promising in terms of future detectability to focus on weak lensing.

We have also shown the Schneider et al. (2012) rescaling of the mass functions with respect to the 'half-mode mass' and found that this resulted in power spectra that matched the $\mathrm{N}$-body simulations well. However, the lack of a prescription for how this rescaling varies with redshift makes it more difficult to use in comparing models to data. It is likely that other prescription for the mass function (e.g. Angulo et al. 2013; Pacucci et al. 2013; Schneider et al. 2013b) will be more appropriate. Also for this reason, the fitting function to the final power spectra found by Viel et al. (2012) describes the redshift evolution of the WDM suppression better.

We have summarised the recent results coming from the Ly $\alpha$ forest data, which still provide the strongest constraints to date, of $3.3 \mathrm{keV}$ at $2 \sigma$ confidence (Viel et al. 2013a). We have summarised forecasts for future large-scale structure surveys, in particular for the Euclid weak lensing survey. This was done in order to show how the free-streaming of WDM, which smoothes out the sub- $0.1 \mathrm{Mpc}$ scales in the linear density field impacts the present day measurements of cosmic shear. These forecasts have indicated that the constraints that could be placed on the $m_{\mathrm{wdm}}$ parameter, i.e. the 'warmth' of DM, from cosmic shear will be comparable, but not stronger, than those coming from $\operatorname{Ly} \alpha$. In other words, because the Ly $\alpha$ forest probes cosmic times in the past that are much closer to the linear regime than today and is a projected measurement of the 3D density field, it has the most constraining power in measuring the small-scale suppression coming from the WDM free-streaming, although the redshift range probed is very different from other observables.

The forecasts for Euclid show that WDM particles with masses of the order of $m_{\mathrm{wdm}} \sim \mathrm{keV}$ have a large enough impact on the non-linear density field to be detectable. It should be noted that interesting constraints on the coldness of $\mathrm{CDM}$ can also be placed by using the properties of individual objects (galaxies, DM haloes, GRBs, etc.) and have been presented by many authors. Future and present surveys like Planck, SDSS and Euclid, SKA, and E-ELT will also help in measuring the small-scale properties of the large-scale structure and place stronger constraints in terms of the mass of the DM particle.

As mentioned above, WDM is not the only model able to alter the $\Lambda \mathrm{CDM}$ cosmology on small scales. It has become clear in the past decade, through the works described in this review, among others, that the present non-excluded models of 'standard' WDM are on the limit of detectability and are, because of the increasingly tighter constraints on them, less able to alleviate the small-scale issues of $\Lambda \mathrm{CDM}$ than initially hoped for. As this review was being written, other authors have started to come to the same conclusions (e.g. Schneider et al. 2013a; Kennedy et al. 2013). Luckily, there remain other types of DM models, alternative to the standard WIMP scenario, for example decaying or self-interacting DM that are also promising and worth to investigate.

\section{ACKNOWLEDGEMENTS}

KM and MV thank their colleagues involved in WDM topics that helped collecting the material for this review and in particular Sarah Bridle, Anže Slosar, Marco Baldi, Jochen Weller, Martin Hahnelt, George Becker, and James Bolton. KM acknowledges support from project TRR 33 'The Dark Universe'. MV is supported by the FP7 ERC Grant 'cosmoIGM' GA-257670 and the INFN/PD51 grant.

\section{REFERENCES}

Aarseth, S. J., \& Fall, S. M. 1980, ApJ, 236, 43

Abazajian, K. 2006, PhRvD, D73, 063513

Abazajian, K., Fuller, G. M., \& Tucker, W. H. 2001, ApJ, 562, 593

Abazajian, K., Switzer, E. R., Dodelson, S., Heitmann, K., \& Habib, S. 2005, PhRvD, D71, 043507

Abell, G. O. 1962, LASP, 8, 335

Amendola, L., et al. 2013, Living Reviews in Relativity, 16, 6

Angulo, R. E., Hahn, O., \& Abel, T. 2013, ArXiv e-prints

Appel, A. 1985, SIAM JSciStatComput, 6, 85

Asaka, T., Blanchet, S., \& Shaposhnikov, M. 2005, PhysLett, B631, 151

Avila-Reese, V., Colin, P., Valenzuela, O., D’Onghia, E., \& Firmani, C. 2001, ApJ, 559, 516

Bagla, J., \& Ray, S. 2003, NewA, 8, 665

Barnes, J., \& Hut, P. 1986, Natur, 324, 446

Bartelmann, M., \& Schneider, P. 2001, PhR, 340, 291

Benitez-Llambay, A., Navarro, J. F., Abadi, M. G., Gottlöber, S., Yepes, G., Hoffman, Y., \& Steinmetz, M. 2013, ApJ, 763, L41

Benson, A. J., Farahi, A., Cole, S., Moustakas, L. A., Jenkins, A., Lovell, M., Kennedy, R., Helly, J., \& Frenk, C. 2013, MNRAS, 428, 1774

Bi, H., \& Davidsen, A. F. 1997, ApJ, 479, 523

Blas, D., Lesgourgues, J., \& Tram, T. 2011, JCAP, 1107, 034

Bode, P., Ostriker, J. P., \& Turok, N. 2001, ApJ, 556, 93

Boehm, C., Mathis, H., Devriendt, J., \& Silk, J. 2005, MNRAS, 360,282 
Bond, J., Efstathiou, G., \& Silk, J. 1980, PhRvL, 45, 1980

Bond, J., \& Szalay, A. 1983, ApJ, 274, 443

Bond, J., Szalay, A., \& Turner, M. S. 1982, PhRvL, 48, 1636

Boyanovsky, D., de Vega, H., \& Sanchez, N. 2008a, PhRvD, D77, 043518

Boyanovsky, D., de Vega, H., \& Sanchez, N. 2008b, PhRvD, D78, 063546

Boyarsky, A., Iakubovskyi, D., \& Ruchayskiy, O. 2012, PhysDarkUnivers, 1, 136

Boyarsky, A., Lesgourgues, J., Ruchayskiy, O., \& Viel, M. 2009a, JCAP, 0905, 012

Boyarsky, A., Lesgourgues, J., Ruchayskiy, O., \& Viel, M. 2009b, PhRvL, 102, 201304

Boyarsky, A., Neronov, A., Ruchayskiy, O., \& Shaposhnikov, M. 2006, JETP Lett, 83, 133

Brooks, A. M., Kuhlen, M., Zolotov, A., \& Hooper, D. 2013, ApJ, 765, 22

Bruns, K., \& Zinnecker, H. 1983, MitAG, 58, 142

Bullock, J. S. 2010, ArXiv e-prints

Burbidge, G. R., \& Sargent, W. L. W. 1969, Comments AstrophysSpacePhys, 1, 220

Busca, N. G. et al. 2013, A\&A, 552, A96

Casarini, L., La Vacca, G., Amendola, L., Bonometto, S. A., \& Maccio, A. V. 2011, JCAP, 1103, 026

Colombi, S., Jaffe, A., Novikov, D., \& Pichon, C. 2009, MNRAS, 393, 511

Cooray, A., Hu, W., \& Miralda-Escude, J. 2000, ApJ, 535, L9

Cooray, A., \& Sheth, R. K. 2002, PhR, 372, 1

Cowsik, R., \& McClelland, J. 1972, PhRvL, 29, 669

Cowsik, R., \& McClelland, J. 1973, ApJ, 180, 7

Croft, R. A. C., Weinberg, D. H., Bolte, M., Burles, S., Hernquist, L., Katz, N., Kirkman, D., \& Tytler, D. 2002, ApJ, 581, 20

Csabai, I. et al. 2003, AJ, 125, 580

Dicke, R. H., Peebles, P. J. E., Roll, P. G., \& Wilkinson, D. T. 1965 , ApJ, 142, 414

Dodelson, S., \& Widrow, L. M. 1994, PhRvL, 72, 17

Donato, F., Gentile, G., Salucci, P., Martins, C. F., Wilkinson, M. et al. 2009, MNRAS, 397, 1169

Dunstan, R. M., Abazajian, K. N., Polisensky, E., \& Ricotti, M. 2011, ArXiv e-prints

Faber, S. M., \& Gallagher, J. S. 1979, ARA\&A, 17, 135

Frigo, M. 1999, SIGPLAN Not, 34, 169

Fuller, G. M., Kusenko, A., Mocioiu, I., \& Pascoli, S. 2003, PhRvD, D68, 103002

Gao, L., \& Theuns, T. 2007, Sci, 317, 1527

Gawiser, E., \& Silk, J. 1998, Sci, 280, 1405

Ghigna, S., Borgani, S., Tucci, M., Bonometto, S. A., Klypin, A., \& Primack, J. R. 1997, ApJ, 479, 580

Green, A. M., Hofmann, S., \& Schwarz, D. J. 2005, JCAP, 8, 3

Gunn, J. E., Lee, B. W., Lerche, I., Schramm, D. N., \& Steigman, G. 1978, ApJ, 223, 1015

Hamann, J., Hannestad, S., Raffelt, G. G., Tamborra, I., \& Wong, Y. Y. 2010, PhRvL, 105, 181301

Hofmann, S., Schwarz, D. J., \& Stöcker, H. 2001, PhRvD, 64, 083507

Hogan, C. J. 1999, ArXiv Astrophysics e-prints

Janák, F. 1958, BAICz, 9, 139

Jenkins, A. et al. 1998, ApJ, 499, 20

Jones, B. J. T., \& Rees, M. J. 1978, in IAU Symposium, Vol. 79, Large Scale Structures in the Universe, ed. M. S. Longair \& J. Einasto (Dordrecht: D. Reidel), 377
Kaiser, N. 1983, ApJ, 273, L17

Katz, N., Weinberg, D. H., \& Hernquist, L. 1996, ApJS, 105, 19

Kennedy, R., Frenk, C., Cole, S., \& Benson, A. 2013, ArXiv e-prints

Klypin, A., Borgani, S., Holtzman, J., \& Primack, J. 1995, ApJ, 444,1

Klypin, A., Holtzman, J., Primack, J., \& Regos, E. 1993, ApJ, 416, 1

Klypin, A., Kravtsov, A. V., Valenzuela, O., \& Prada, F. 1999, ApJ, 522, 82

Knebe, A., Devriendt, J. E., Gibson, B. K., \& Silk, J. 2003, MNRAS, 345,1285

Kusenko, A. 2009, PhR, 481, 1

Laine, M., \& Shaposhnikov, M. 2008, JCAP, 0806, 031

Lesgourgues, J. 2011, ArXiv e-prints

Lesgourgues, J., \& Tram, T. 2011, JCAP, 9, 32

Lesgourgues, J., Viel, M., Haehnelt, M. G., \& Massey, R. 2007, JCAP, 11,8

Lewis, A., Challinor, A., \& Lasenby, A. 2000, ApJ, 538, 473

Limber, D. N. 1953, ApJ, 117, 134

Lin, D. N. C., \& Faber, S. M. 1983, ApJ, 266, L21

Lovell, M. R., Eke, V., Frenk, C. S., Gao, L., Jenkins, A. et al. 2012, MNRAS, 420, 2318

Lovell, M. R., Frenk, C. S., Eke, V. R., Jenkins, A., Gao, L., \& Theuns, T. 2013, ArXiv e-prints

LoVerde, M., \& Afshordi, N. 2008, PhRvD, D78, 123506

Ma, C.-P., \& Bertschinger, E. 1995, ApJ, 455, 7

Maccio, A. V., Paduroiu, S., Anderhalden, D., Schneider, A., \& Moore, B. 2012, MNRAS, 424, 1105

Madsen, J., \& Epstein, R. I. 1984, ApJ, 282, 11

Markovič, K., Bridle, S., Slosar, A., \& Weller, J. 2011, JCAP, 1101, 022

McDonald, P. et al. 2005, ApJ, 635, 761

Meiksin, A. A. 2009, RvMP, 81, 1405

Narayanan, V. K., Spergel, D. N., Davé, R., \& Ma, C.-P. 2000, ApJ, 543, L103

Navarro, J. F., Frenk, C. S., \& White, S. D. 1997, ApJ, 490, 493

Nolthenius, R., Klypin, A., \& Primack, J. R. 1994, ApJ, 422, L45

Olive, K. A., Steigman, G., \& Walker, T. P. 2000, PhR, 333, 389

Paal, G. 1976, AN, 297, 311

Pacucci, F., Mesinger, A., \& Haiman, Z. 2013, ArXiv e-prints

Pagels, H., \& Primack, J. R. 1982, PhRvL, 48, 223

Palanque-Delabrouille, N., et al. 2013, ArXiv e-prints

Peebles, P. 1982, ApJ, 258, 415

Penzias, A. A., \& Wilson, R. W. 1965, ApJ, 142, 419

Petraki, K., \& Kusenko, A. 2008, PhRvD, D77, 065014

Planck Collaboration, et al. 2013, ArXiv e-prints

Press, W. H., \& Schechter, P. 1974, ApJ, 187, 425

Primack, J. R. 1997, ArXiv Astrophysics e-prints

Primack, J. R. 2003, NuclPhyBProcSuppl, 124, 3

Primack, J. R., \& Gross, M. A. K. 1998, ArXiv Astrophysics e-prints

Primack, J. R., Holtzman, J., Klypin, A., \& Caldwell, D. O. 1995, PhRvL, 74, 2160

Refregier, A., Amara, A., Kitching, T., Rassat, A., Scaramella, R., et al. 2010, ArXiv e-prints

Reid, I. N. et al. 1991, PASP, 103, 661

Rudnicki, K. 1976, AN, 297, 317

Schaye, J. et al. 2010, MNRAS, 402, 1536

Schneider, A., Anderhalden, D., Maccio, A., \& Diemand, J. 2013a, ArXiv e-prints

Schneider, A., Smith, R. E., Maccio, A. V., \& Moore, B. 2012, MNRAS, 424, 684 
Schneider, A., Smith, R. E., \& Reed, D. 2013b, MNRAS, 433, 1573

Schramm, D. N., \& Steigman, G. 1981, ApJ, 243, 1

Schramm, D. N., \& Wagoner, R. V. 1977, AnnuRevNuclParticleSci, 27,37

Schwarzschild, M. 1954, AJ, 59, 273

Sciama, D. W. 1983, in IAU Symposium, Vol. 104, Early Evolution of the Universe and its Present Structure, ed. G. O. Abell \& G. Chincarini (Dordrecht: Springer), 493

Seljak, U. 2000, MNRAS, 318, 203

Seljak, U., Makarov, A., McDonald, P., \& Trac, H. 2006a, PhRvL, 97, 191303

Seljak, U., Slosar, A., \& McDonald, P. 2006b, JCAP, 0610, 014

Seljak, U., \& Zaldarriaga, M. 1996, ApJ, 469, 437

Semboloni, E., Hoekstra, H., Schaye, J., van Daalen, M. P., \& McCarthy, I. J. 2011, MNRAS, 417, 2020

Semenov, V., Pilipenko, S., Doroshkevich, A., Lukash, V., \& Mikheeva, E. 2013, ArXiv e-prints

Shafi, Q., \& Stecker, F. W. 1984, PhRvL, 53, 1292

Shao, S., Gao, L., Theuns, T., \& Frenk, C. S. 2013, MNRAS, 430, 2346

Sheth, R. K., \& Tormen, G. 1999, MNRAS, 308, 119

Sitwell, M., Mesinger, A., Ma, Y.-Z., \& Sigurdson, K. 2013, ArXiv e-prints

Slosar, A. et al. 2013, JCAP, 4, 26

Smail, I., Ellis, R. S., \& Fitchett, M. J. 1994, MNRAS, 270, 245

Smith, R. et al. 2003, MNRAS, 341, 1311

Smith, R. E., \& Markovič, K. 2011, PhRvD, D84, 063507

Sommer-Larsen, J., \& Dolgov, A. 2001, ApJ, 551, 608

Springel, V. 2005, MNRAS, 364, 1105

Springel, V., Yoshida, N., \& White, S. D. 2001, NewA, 6, 79

Stecker, F. W., \& Shafi, Q. 1983, PhRvL, 50, 928

Takada, M., \& Jain, B. 2004, MNRAS, 348, 897

Tikhonov, A. V., Gottlöber, S., Yepes, G., \& Hoffman, Y. 2009, MNRAS, 399, 1611

Tremaine, S., \& Gunn, J. E. 1979, PhRvL, 42, 407 van Daalen, M. P., Schaye, J., Booth, C., \& Vecchia, C. D. 2011, MNRAS, 415, 3649

Viel, M., Becker, G. D., Bolton, J. S., \& Haehnelt, M. G. 2013a, ArXiv e-prints

Viel, M., Becker, G. D., Bolton, J. S., Haehnelt, M. G., Rauch, M., \& Sargent, W. L. W. 2008, Physical Review Letters, 100, 041304

Viel, M., Haehnelt, M. G., \& Lewis, A. 2006, MNRAS, 370, L51

Viel, M., Haehnelt, M. G., \& Springel, V. 2004, MNRAS, 354, 684

Viel, M., Lesgourgues, J., Haehnelt, M. G., Matarrese, S., \& Riotto, A. 2005, PhRvD, D71, 063534

Viel, M., Lesgourgues, J., Haehnelt, M. G., Matarrese, S., \& Riotto, A. 2006, PhRvL, 97, 071301

Viel, M., Markovič, K., Baldi, M., \& Weller, J. 2012, MNRAS, 421, 50

Viel, M., Matarrese, S., Mo, H. J., Haehnelt, M. G., \& Theuns, T. 2002, MNRAS, 329, 848

Viel, M., Schaye, J., \& Booth, C. M. 2013b, MNRAS, 429, 1734

Vinas, J., Salvador-Sole, E., \& Manrique, A. 2012, MNRAS, 424, L6

Wang, J., \& White, S. D. M. 2007, MNRAS, 380, 93

Weinberg, D. H., Bullock, J. S., Governato, F., Kuzio de Naray, R., \& Peter, A. H. G. 2013, ArXiv e-prints

Wesson, P. S. 1978, ApL, 19, 127

White, S. D. M., \& Frenk, C. S. 1991, ApJ, 379, 52

White, S. D. M., Frenk, C. S., Davis, M., \& Efstathiou, G. 1987, ApJ, 313, 505

White, S. D. M., \& Negroponte, J. 1982, MNRAS, 201, 401

White, S. D. M., \& Rees, M. J. 1978, MNRAS, 183, 341

Zavala, J., Jing, Y. P., Faltenbacher, A., Yepes, G., Hoffman, Y., Gottlöber, S., \& Catinella, B. 2009, ApJ, 700, 1779

Zeldovich, Y. 1970, A\&A, 5, 84

Zentner, A. R., \& Bullock, J. S. 2003, ApJ, 598, 49

Zwicky, F. 1937, ApJ, 86, 217 